Submitted to the Annals of Applied Statistics

arXiv: XXXXXXXXYYYYYYZZZZZZZ

\title{
UNSUPERVISED STREAMING ANOMALY DETECTION FOR INSTRUMENTED INFRASTRUCTURE
}

\author{
By Henrique Hoeltgebaum ${ }^{\S, \uparrow, \dagger, *}$, Niall Adams ${ }^{\S, \|}$, And F. \\ Din-Houn LAU ${ }^{\S, * *}$ \\ Department of Mathematics, Imperial College London ${ }^{\S}$, Pontifical \\ Catholic University of Rio de Janeiro " and Data Science Institute, \\ Imperial College London ", The Alan Turing Institute**
}

\begin{abstract}
Structural Health Monitoring (SHM) often involves instrumenting structures with distributed sensor networks. These networks typically provide high frequency data describing the spatio-temporal behaviour of the assets. A main objective of SHM is to reason about changes in structures' behaviour using sensor data. We construct a streaming anomaly detection method for data from a railway bridge instrumented with a fibre-optic sensor network. The data exhibits trend over time, which may be partially attributable to environmental factors, calling for temporally adaptive estimation. Exploiting a latent structure present in the data motivates a quantity of interest for anomaly detection. This quantity is estimated sequentially and adaptively using a new formulation of streaming Principal Component Analysis. Anomaly detection for this quantity is then provided using Conformal Prediction. Like all streaming methods, the proposed method has free control parameters which are set using simulations based on bridge data. Experiments demonstrate that this method can operate at the sampling frequency of the data while providing accurate tracking of the target quantity. Further, the anomaly detection is able to detect train passage events. Finally the method reveals a previously unreported cyclic structure present in the data.
\end{abstract}

1. Introduction. Modern civil engineering is increasingly leveraging sensor technology to understand and monitor physical assets, such as bridges and pipe networks (Butler et al., 2016a, 2018). This sensor technology is becoming cheaper to deploy and hence more widely used. The ultimate ambition for such sensor networks is to better understand the behaviour and degradation of physical assets. However, there are significant data processing

\footnotetext{
${ }^{*}$ Corresponding author.

${ }^{\dagger}$ Supported by the National Council for Research and Development, CNPq, Ministry of Science and Technology, Brazil.

** Supported by The Lloyds Register Foundation Programme on Data-Centric Engineering, The Alan Turing Institute

Keywords and phrases: Structural Health Monitoring, streaming PCA, stochastic gradient descent, adaptive estimation, conformal prediction.
} 
and analysis challenges prior to this.

The concern of this paper is the analysis of sensor data from railway bridges instrumented with a fibre-optic sensor network. The sensor system is used to quantify the stress behaviour of the asset, typically at high frequency. The sensor network is distributed spatially over the bridge and in some sense captures spatio-temporal behaviour.

We seek to develop a streaming method for monitoring the bridge, as a whole, for train passage events and other events which manifest similar responses. This is challenging for a number of reasons. First, the data is recorded at high frequency, often $250 \mathrm{~Hz}$. Constructing sequential methods that can update at this rate is challenging. Second, the data is subject to unpredictable temporal drift, for example relating to temperature, and other less understood processes. This calls for temporally adaptive methodology. Third, since the sensor system is physically distributed, it provides a view of the whole bridge at every time instance (hereafter, tick). A train passage event has a direction with relation to the sensors and hence manifests differently over the network at any tick. Analysing sensors separately will lead to definitional problems (e.g. when did the event start) and issues of multiple testing.

In this paper we propose a novel streaming methodology intended to identify events that are departures from the "at-rest" state of the entire bridge. During this "at-rest" state - when a train is not interacting with the bridge - exploratory data analysis reveals a striking low dimensional latent structure arising from the sensor network. Further, this latent structure is a linear combination of all sensors in the network, and hence represents the collective behaviour of the bridge. We develop a streaming estimation procedure for tracking components of this structure at data rate, by which we mean the algorithmic update procedure is faster than the frequency of observation. The sensor network records train passage events as a complicated spatiotemporal response. Precisely labelling the start and end of these events is difficult, because the first sensor in the direction of travel will respond earlier than the last sensor. Moreover, the sensor system exhibits a reaction before and after the train is on the bridge. The anomaly detector is designed to capture the change in latent structure from at-rest to train passage event. We are therefore proposing an unsupervised anomaly detection approach intended to detect specific collective departures from at-rest behaviour.

The structure of this paper is as follows. Section 2 provides a detailed overview of the bridge sensor system and a specific data set. Principal Component Analysis (PCA) reveals a specific low dimensional latent structure during at-rest periods. In Section 3 a streaming PCA procedure is devel- 
oped. This procedure synthesises ideas of adaptive estimation (Haykin, 2008) with ideas for sequential eigendecomposition (see Cardot and Degras (2018); Balzano et al. (2018) and references therein). A collection of numerical problems arise with sequential eigendecomposition, which we address. Additionally, like all streaming estimation methods a number of input parameters need to be set. In Section 4 we use ideas from Conformal Prediction (Vovk et al., 2005; Vovk, 2013) to deal with the challenge of statistical anomaly detection for eigendecomposition. The detection performance of the method relates to its estimation performance, which in turn depends on input parameters, as noted above. Section 5 reports a simulation study designed to both compare variants of the methodology and determine input parameters for practical deployment. Finally, the preferred methodology is deployed against a large amount of bridge data in Section 6. In addition to demonstrating the detection capabilities of the methodology, the proposed method also reveals interesting and previously unreported properties of the sensor system.

2. Instrumented Infrastructure and Data. In this section, a brief review of Structure Health Monitoring (SHM) and a detailed description of the bridge data set are provided.

2.1. Structural Health Monitoring. The area of SHM has changed in recent times due to the automated collection of data pertaining to physical assets. Historically and currently most integrity evaluation is performed on a manual basis, which is costly and time-consuming. Structures such as railway bridges and pipe networks are now being instrumented with a variety of sensor systems in an effort to better understand their behaviour and reduce the cost burden of monitoring (Farrar and Worden, 2006; Das et al., 2016; Todd et al., 2006; Bowers et al., 2016; Butler et al., 2016b; Glisic et al., 2005; Lau et al., 2018; Measures et al., 1992; Hernandez-Garcia and Masri, 2014). Asset operators are using these data to reason about SHM questions. There are significant challenges related to the development of statistical methods for handling such data. Physical assets have both spatial and temporal extent and the data exhibits rich idiosyncratic structure which raises challenges for spatio-temporal modelling. The high frequency recording rate of the data poses a challenge for data curation and statistical analysis. Detection of train passage events at data rate requires both acquiring the data and performing a statistical procedure. Some of these statistical aspects have been addressed by Lau et al. (2018). Data generated by the sensor system is a combination of physical response of the bridge, noise arising from the sensor system and environmental factors at each sensor location. 
2.2. Fiber Bragg Grating sensors. Fiber Bragg Grating (FBG) sensors are commonly used to measure strain, that is, for example, the vertical deflection of a bridge under load. In this work, the sensor system consists of a distributed network of fibre-optic strain sensors. These sensors used inscribed Bragg Gratings (Micron Optics, 2013) within the fibre-optic cable which refract light at a particular wavelength. When subjected to strain, the cable is deformed resulting in a change of wavelength.

A statistical challenge arises because the FBG cable, and hence data, also responds to environmental factors such as temperature changes. This is just one of several possible factors that contribute to temporal variations observed in the data. A sophisticated algorithm is used to transform the refracted light in the optical cable to wavelength (more details in Micron Optics (2013)).

The raw data is measured in wavelength (nano-meters), although engineers prefer the physical quantity, strain. By construction, each sensor's wavelength is offset by a fixed constant. Wavelength and strain are linearly related and hence we will report results on the wavelength scale. Denote the wavelength measurement at tick $N$ from sensor $j$ as $x_{j, N}$ for which we form the vector over $d$ sensors

$$
\mathbf{x}_{N}=\left(x_{1, N}, \ldots, x_{d, N}\right) .
$$

2.3. Data. We are concerned with data from a steel-concrete railway bridge located in Staffordshire, UK. This bridge is a 26.8 metre composite instrumented with 80 FBG sensors. In this case, the sensor network was installed during construction, though retrofitting is possible. The acquisition rate of the data is $250 \mathrm{~Hz}$, i.e., each sensor records 250 ticks a second. The sensors are organised as in Figure 1, spaced one metre along the fibre-optic cable. There are four cables located in the main girders, which are each 20 metres long.

Examining data from an individual sensor reveals unusual structures. For example, Figure 2 presents a sequence of observations from a single sensor which exhibits a "banding" structure. This is an artefact of the sensor recording algorithm mentioned above. Note Figure 2 displays around 8 seconds of data which exhibits little temporal variation over this short period. However, Figure 3 presents a smoothed version of multiple sensors over a much longer period. This shows that there is temporal variation, or drift, which is different by sensor. This drift motivates the use of adaptive estimation in the proposed methodology. Figure 4 presents measurements from multiple sensors including a train passage event. Note that sensor measurements during the event differ according to their spatial location. 


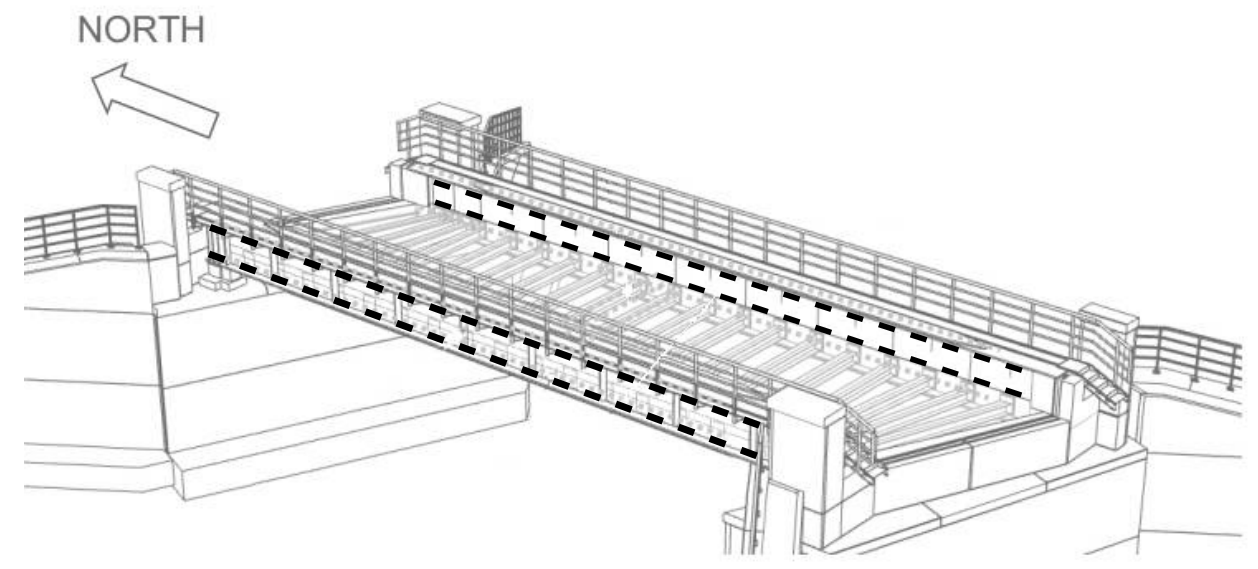

Fig 1: Configuration of sensor cables (in black dotted lines) along the bridge. Each of the four sensors contains 20 fibre Bragg gratings.

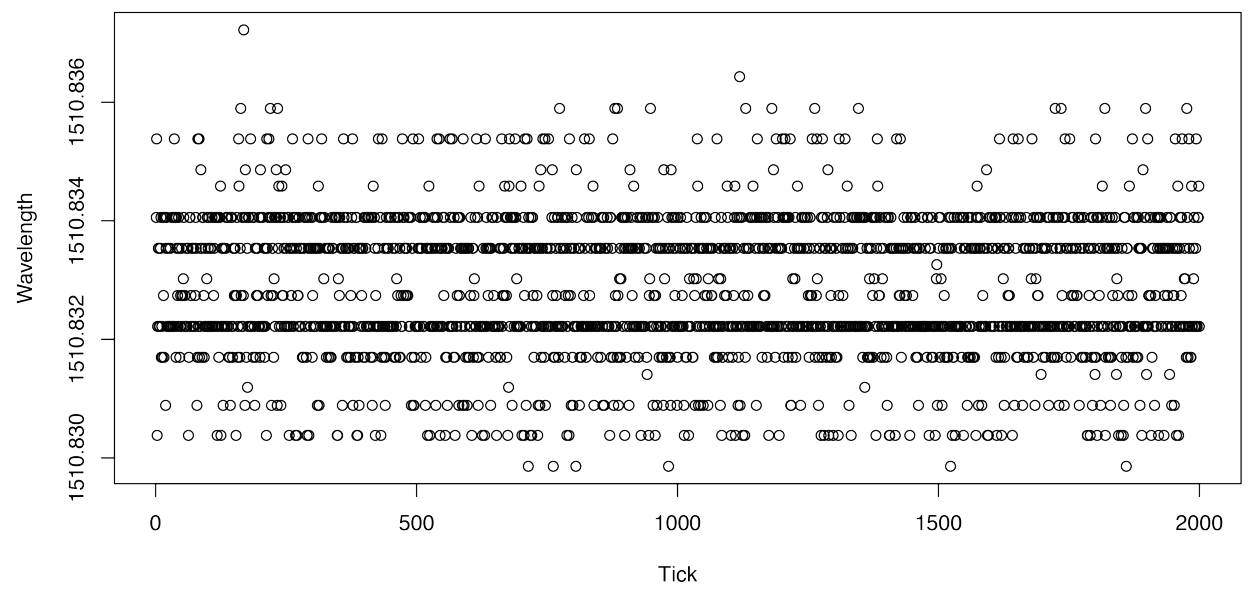

Fig 2: Wavelength measurements of a single sensor during an at-rest period.

It is important to distinguish the two types of periods present in the data: periods at-rest and train passage events. Both types capture the space-time response of the sensor system, however at-rest it is reasonable to assume that the variation in the sensor system is simply noise. Identifying train passage events is a step toward SHM, since changes in response during train passage events might indicate degradation. 


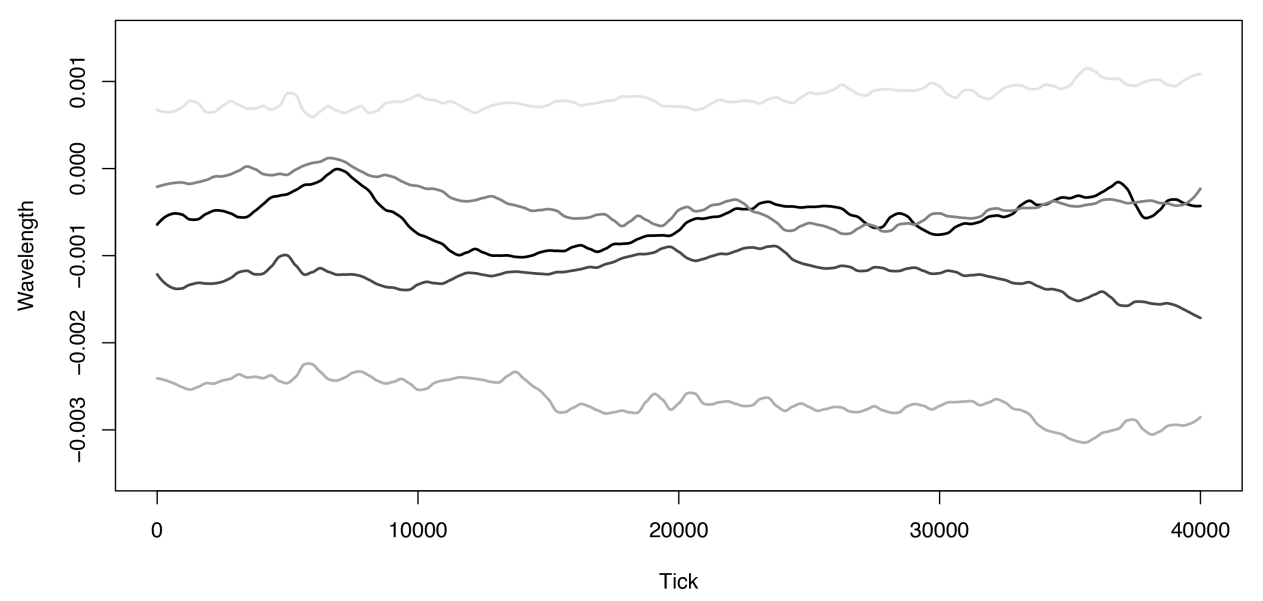

Fig 3: Smoothed (using LOESS (Cleveland, 1979)) versions of five sensors during an at-rest period. For illustration, the first value of each sensor was subtracted from each stream.

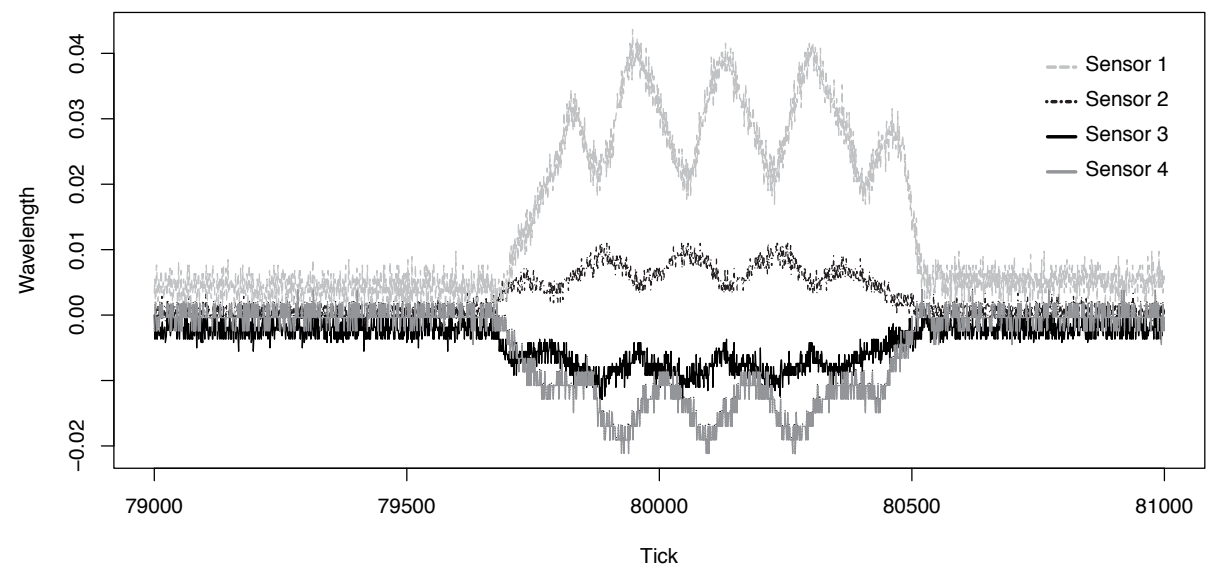

Fig 4: Measurement for four sensors including a train passage event. For illustration, the first value of each sensor was subtracted from each stream.

The collective response of the sensor system can be understood using various statistical procedures. In this case Principal Component Analysis (PCA), as used in Jolliffe (2011), reveals an unexpected structure. Using PCA on an at-rest period of 5000 ticks of data, we find that the first 2 
principal components account for nearly $40 \%$ of the variation in the data. The scores of PCA are computed as

$$
T_{q}=\mathbf{U}_{q} X
$$

where $X$ is a centred data matrix and $\mathbf{U}_{q}=\left[\mathbf{u}_{1} \ldots \mathbf{u}_{q}\right]$ is the loading matrix consisting of the eigenvectors of the first $q$ principal components. Denote the covariance matrix of $X$ as $\Sigma$ and the eigenvalue corresponding to eigenvector $\mathbf{u}_{j}$ as $\gamma_{j}$. Figure 5a shows that the scores of the first $q=2$ principal components scores exhibit an annular structure. In fact, this latent structure is observed for all at-rest periods in this particular data and we observe it for other FBG instrumented bridges. This annular structure is seldom observed in multivariate data analysis, although it has been noted on occasion. For example, related sinusoidal patterns have been observed in population genetics studies, in explorations of spatially distributed populations (Novembre and Stephens, 2008). In temporal studies of gene expression, circular principal components have been observed (Scholz, 2007). At present, we are unable to provide a compelling explanation for the presence of this structure in the bridge data, from either a mathematical or physical point of view, and hence prefer not to speculate beyond attributing the phenomena to spatiotemporal characteristics of the sensor network.

As far as we are aware, this latent structure is not fully appreciated in the SHM community. Again, we stress that this structure does not characterise the physics of the bridge but rather the innate properties of the sensor system. The train passage event has a marked effect on this latent structure, see Figure 5b. More variation is attributed to the first component rather than being shared approximately equally across the first two. This property is observed over many data examples and is exactly this difference that we exploit to construct an anomaly detection system concerned with the collective spatio-temporal response of the bridge.

Figure 6 is a schematic that shows an illustrative data stream from a single sensor during an at-rest period, followed by a train passage event, followed by another at-rest period. The tick in which the train passage event starts is denoted by $J_{S}$ while $J_{E}$ denotes the end of the event. Our anomaly detection system will attempt to detect $J_{S}$. This detection is performed by looking for departures away from the collective at-rest periods. In practice we would want to identify the whole event but it is more convenient to simply collect a fixed amount of data, $\kappa$, following the detection. These blocks of data would then be subject to further offline analysis. The period $\delta$ is an allowance for measuring the detection delay, see Section 4. Detection problems of this type raise the unresolved question of whether to continue estimation following 


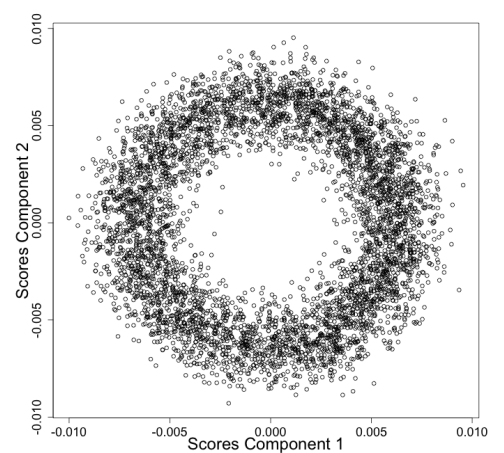

(a)

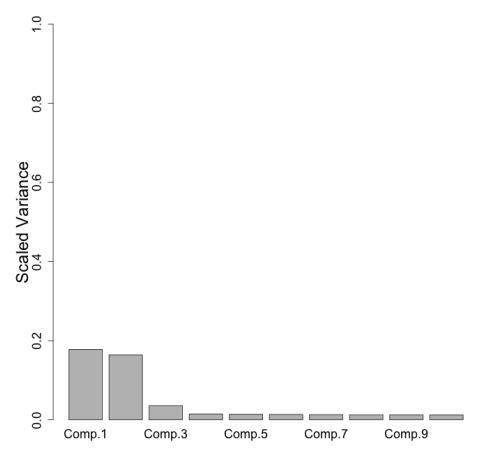

(c)

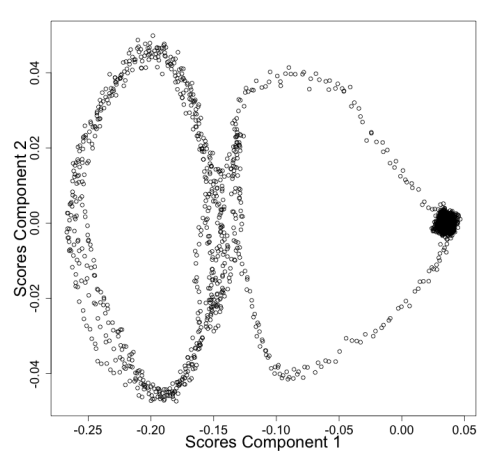

(b)

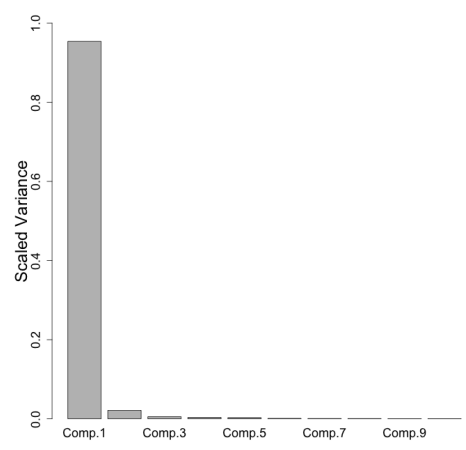

(d)

Fig 5: PCA result for the bridge data. (a): First two scores during atrest period based on a batch of 5000 measurements. (b): First two scores, computed using a sliding window of 5000 measurements including a train passage event. The left "lobe" corresponds to at-rest periods. (c): Scaled screeplot during at-rest period. (d): Scaled screeplot during train passage event.

detection or to restart. This is addressed further in Section 5.

3. Streaming PCA. Computing PCA on batches of data is generally straightforward. However, computing PCA sequentially and with some capacity for temporal adaption is challenging, as described in the conclusions of Cardot and Degras (2018). As a matter of fact, this is the first work to derive a time-dependent adaptive forgetting factor mechanism that sequentially tracks aspects of PCA in a streaming data setting. We develop 


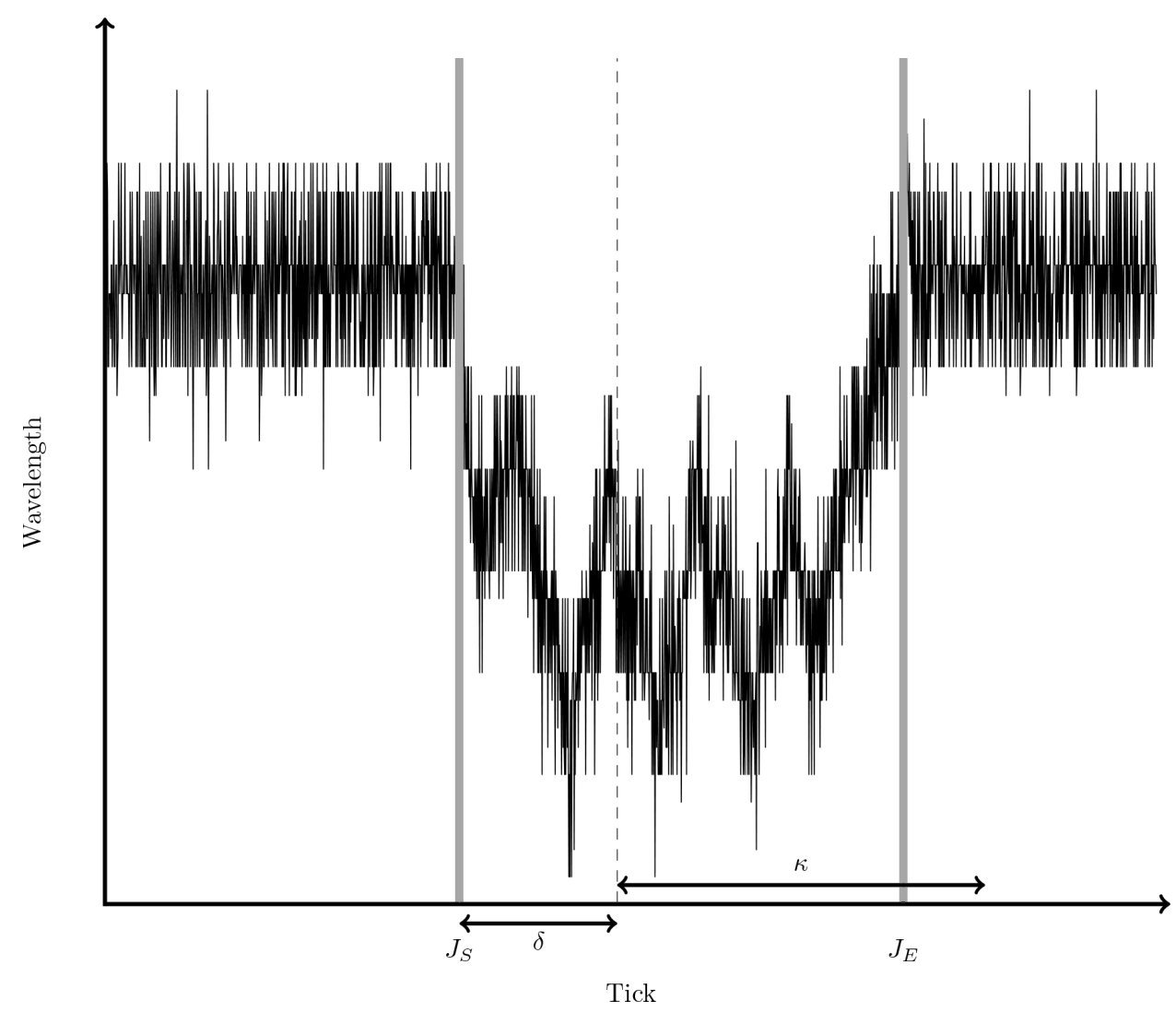

Fig 6: Illustration of detection when the train passage event happens. The events starts at tick $J_{S}$ and ends at tick $J_{E}$. We aim to capture the event between $J_{S}$ and the tolerance period $\delta$, which we deem correct detection in simulation studies.

a sequential estimation method in Section 3.2 through the use of adaptive filtering techniques and extend it to a streaming PCA context in Section 3.3 .

3.1. Principal Component Analysis. Denote $X \in \mathbb{R}^{N \times d}$ as a columncentred data matrix whose rows consists of $\mathbf{x}_{1}, \ldots, \mathbf{x}_{N}$, where $\mathbf{x}_{i}$ is the $d$ dimensional multivariate sensor measurement at tick $i$. This centering is achieved using the mean vector $\boldsymbol{\mu}=\left[\mu_{1}, \ldots, \mu_{d}\right]$ where $\mu_{j}=\frac{1}{N} \sum_{i=1}^{N} X_{i, j}$. Our methodology will later reformulate this estimator. PCA seeks an accurate representation of the original data set in a lower-dimensional subspace $\mathbb{R}^{q}, q<d$, which maximizes the explained variance. PCA seeks a projection 
matrix $\hat{\mathbf{U}} \hat{\mathbf{U}}^{T}$ which is approximated by

$$
\hat{\mathbf{U}}=\underset{\mathbf{U} \in \mathbb{R}^{d \times q}, \mathbf{U}^{T} \mathbf{U}=I_{q}}{\operatorname{argmin}}\left\|X-X \mathbf{U U}^{T}\right\|_{F}^{2}
$$

where $\|\cdot\|_{F}$ denotes the Frobenius norm, $I_{q}$ denotes the $q$ dimensional identity matrix and $\hat{\mathbf{U}}=\left[\hat{\mathbf{u}}_{1}, \ldots, \hat{\mathbf{u}}_{q}\right]$ are the $q$ largest eigenvectors of the sample covariance matrix $\Sigma$. These largest $q$ eigenvectors, associated with the $q$ leading eigenvalues $\gamma_{(1)} \geq \gamma_{(2)} \geq \ldots \geq \gamma_{(d)}$, are the principal components. For reasons explained in the previous section, we are interested in $q=2$.

A streaming PCA framework needs both a sequential updating mechanism and a method for reweighting estimators as the data process changes. Recalling the quantities involved in the eigendecomposition, sequential updates are required for the mean vector $\boldsymbol{\mu}$, to achieve centering, and the covariance matrix $\Sigma$. To accommodate trend and changes in the covariance structure over time, we require time-dependent estimators, $\boldsymbol{\mu}_{N}$ and $\Sigma_{N}$. The following sections outline the construction of adaptive and sequential estimates of $\boldsymbol{\mu}_{N}$ and $\Sigma_{N}$ and the subsequent sequential construction of the eigendecomposition. In this latter step, there is some scope for reducing computational burden by evaluating a partial eigendecomposition.

3.2. Adaptive Estimation. Adaptive filtering (Haykin, 2008) provides suitable tools for both sequential and time-dependent estimation of $\boldsymbol{\mu}_{N}$ and $\Sigma_{N}$. In practice, when dealing with streaming data, methods need to cope with (i) memory efficiency, i.e., the entire data set cannot be stored, and (ii) sequential estimation of model parameters (Aggarwal, 2007; Gama, 2010; Benczúr et al., 2018). Temporal adaptation is provided by incorporating a forgetting factor $(\mathrm{FF})$, that controls the contribution of each data point to the estimator. Practically setting this FF parameter, $\lambda \in(0,1)$, is challenging in a streaming data context. Therefore much interest has focused on sequentially selecting an adaptive forgetting factor $(\mathrm{AFF})-\lambda_{N}$, using an updating mechanism based on stochastic gradient descent (Haykin, 2008; Anagnostopoulos et al., 2012; Bodenham and Adams, 2017).

Consider the univariate data stream

$$
\left\langle y_{1}, y_{2}, \ldots, y_{N-1}, y_{N}, \ldots\right\rangle,
$$

from which the objective is to accurately estimate $E\left[Y_{j}\right]$ sequentially at each tick $j$. If $E\left[Y_{j}\right]$ is the same constant for all $j$ then the sample mean

$$
\bar{y}_{j}=\frac{1}{j} \sum_{i=1}^{j} y_{i},
$$


is a sensible estimate which admits a sequential formulation. On the other hand, if $E\left[Y_{j}\right]$ varies over $j$, then the estimate would be inappropriate. This limitation motivates the use of adaptive estimation to calculate the mean at time $N$, in which more weight is placed on more recent measurements. These methods result in improved estimation for time-varying processes (Bodenham and Adams, 2017). A fixed FF $\lambda$ is introduced into Equation (3.2) with normalizing constant $\left(w_{N, \lambda}\right)$ (sometimes called the effective sample size) to weight the estimation process as follows

$$
\bar{y}_{N, \lambda}=\frac{1}{w_{N, \lambda}} \sum_{i=1}^{N} \lambda^{N-i} y_{i}, \quad w_{N, \lambda}=\sum_{i=1}^{N} \lambda^{N-i} .
$$

This formulation leads to a sequential computation for streaming contexts by defining the following updating mechanism for $N \geq 1$,

$$
\begin{aligned}
m_{N+1, \lambda} & =\lambda m_{N, \lambda}+y_{N+1} \\
w_{N+1, \lambda} & =\lambda w_{N, \lambda}+1 \\
\bar{y}_{N, \lambda} & =\frac{m_{N, \lambda}}{w_{N, \lambda}},
\end{aligned}
$$

with $m_{1, \lambda}=y_{1}$ and $w_{1, \lambda}=1$. Setting $\lambda=0$ corresponds to forgetting all previous measurements and only using the most recent measurement, i.e. $\bar{y}_{N, \lambda}=y_{N}$. On the other hand, $\lambda=1$ corresponds to no forgetting, and then the forgetting factor mean, $\bar{y}_{N, \lambda}$, is simply the arithmetic mean given in Equation (3.2).

A more flexible approach is based on AFF, which results in a sequence of FFs $\vec{\lambda}=\left(\lambda_{1}, \ldots, \lambda_{N}\right)$ over time. As shown later this sequence can be selected using sequential stochastic gradient descent (SGD) approaches. Practical algorithms restrict the range of $\lambda$ to prevent it becoming too small, see for example Bodenham and Adams (2017). We use these adaptive filtering techniques to update $\boldsymbol{\mu}_{N}$ and $\Sigma_{N}$ in the eigendecomposition as described next.

Consider the following system of sequential update equations for a mean vector

$$
\begin{aligned}
\mathbf{m}_{N+1, \vec{\lambda}} & =\lambda_{N} \mathbf{m}_{N, \vec{\lambda}}+\mathbf{x}_{N+1} \\
w_{N+1, \vec{\lambda}} & =\lambda_{N} w_{N, \vec{\lambda}}+1 \\
\boldsymbol{\mu}_{N, \vec{\lambda}} & =w_{N, \vec{\lambda}}^{-1} \mathbf{m}_{N, \vec{\lambda}},
\end{aligned}
$$

with $\mathbf{m}_{1, \vec{\lambda}}=\mathbf{x}_{1}$ and $w_{1, \vec{\lambda}}=1$. As pointed out by Bodenham and Adams (2017) and shown in Appendix 8.1, it is possible to rewrite Equation (3.8) 
as

$$
\boldsymbol{\mu}_{N+1, \vec{\lambda}}=\left[1-w_{N+1, \vec{\lambda}}^{-1}\right] \boldsymbol{\mu}_{N, \vec{\lambda}}+\left[w_{N+1, \vec{\lambda}}^{-1}\right] \mathbf{x}_{N+1},
$$

which, following further manipulation, results in the following updating mechanism for the covariance matrix

$$
\begin{aligned}
\Sigma_{N+1, \vec{\lambda}} & =\left[1-w_{N+1, \vec{\lambda}}^{-1}\right] \Sigma_{N, \vec{\lambda}} \\
& +\left[w_{N+1, \vec{\lambda}}^{-1}\right]\left(\mathbf{x}_{N+1}-\boldsymbol{\mu}_{N+1, \vec{\lambda}}\right)\left(\mathbf{x}_{N+1}-\boldsymbol{\mu}_{N+1, \vec{\lambda}}\right)^{T},
\end{aligned}
$$

with $\boldsymbol{\mu}_{1, \vec{\lambda}}=\mathbf{x}_{1}$ and $\Sigma_{1, \vec{\lambda}}=I_{d}$.

The fixed forgetting version of updates (3.9) and (3.10) are familiar in the streaming PCA literature (see Cardot and Degras (2018); Balzano et al. (2018) and references therein).

To select $\lambda_{N}$, we use SGD which requires a cost function, $L_{N+1, \vec{\lambda}}$. The update of $\lambda_{N}$ is defined as

$$
\lambda_{N+1}=\lambda_{N}-\eta \frac{\partial}{\partial \vec{\lambda}} L_{N+1, \vec{\lambda}}
$$

where $\eta$ is the step size and $\lambda_{1}=1$. We will set the step size based on performance in realistic simulations experiments.

In this paper, similar to Bodenham and Adams (2017), the cost function is

$$
L_{N+1, \vec{\lambda}}=\left[\boldsymbol{\mu}_{N, \vec{\lambda}}-\mathbf{x}_{N+1}\right]^{T}\left[\boldsymbol{\mu}_{N, \vec{\lambda}}-\mathbf{x}_{N+1}\right] .
$$

There is some flexibility in the choice of cost function. Anagnostopoulos et al. (2012) shows that differentiable and likelihood-based cost functions yield efficient update equations for the exponential family of distributions. For example, Equation (3.9) can be motivated by an i.i.d. Gaussian argument.

The derivative in (3.11) depends on time-varying quantities which are evident after some manipulation, resulting in

$$
\underset{\partial \vec{\lambda}}{\vec{\partial}} L_{N+1, \vec{\lambda}}=2\left[\frac{\partial}{\partial \vec{\lambda}} \boldsymbol{\mu}_{N, \vec{\lambda}}\right]^{T}\left[\boldsymbol{\mu}_{N, \vec{\lambda}}-\mathbf{x}_{N+1}\right] \text {. }
$$

Bodenham and Adams (2017) compute $\frac{\partial}{\partial \vec{\lambda}} \boldsymbol{\mu}_{N, \vec{\lambda}}$ from first principles. From their computation

$$
\underset{\partial \vec{\lambda}}{\stackrel{\partial}{\vec{\lambda}}} \boldsymbol{\mu}_{N, \vec{\lambda}}=\left[\boldsymbol{\Delta}_{N, \vec{\lambda}} w_{N, \vec{\lambda}}-\mathbf{m}_{N, \vec{\lambda}} \Omega_{N, \vec{\lambda}}\right] / w_{N, \vec{\lambda}}^{2}
$$


the following two auxiliary quantities appear

$$
\begin{aligned}
& \Omega_{N+1, \vec{\lambda}}=\lambda_{N} \Omega_{N, \vec{\lambda}}+w_{N+1, \vec{\lambda}}, \\
& \Delta_{N+1, \vec{\lambda}}=\lambda_{N} \Delta_{N, \vec{\lambda}}+\mathbf{m}_{N+1, \vec{\lambda}},
\end{aligned}
$$

which must also be updated sequentially. Note that $\Delta_{N, \vec{\lambda}}$ is a vector and $\Omega_{N, \vec{\lambda}}$ is a scalar valued quantity where $\Omega_{1, \vec{\lambda}}=1$ and $\Delta_{1, \vec{\lambda}}=\mathbf{x}_{1}$.

This section has outlined a complete sequential updating mechanism for estimating the mean vector and covariance matrix for a time-varying process. We now turn to the construction of principal components using these timevarying estimates.

3.3. Streaming PCA. A naive approach would evaluate the full eigendecomposition of $\Sigma_{N}$ or the Singular Value Decomposition (SVD). However for streaming problems this will be computationally burdensome at each tick. A number of approaches for sequentially updating an eigendecomposition have been proposed (Sanger, 1989; Weng et al., 2003; Mitliagkas et al., 2013; Warmuth and Kuzmin, 2008; Boutsidis et al., 2015). In the case of a known covariance matrix $\Psi$, Oja and Karhunen (1985); Oja (1992), proposed a gradient ascent update for the full projection matrix, previously defined in Equation (3.1) for the batch case, of the form

$$
\mathbf{U}_{N+1}=\mathbf{U}_{N}+\xi_{N} \Psi \mathbf{U}_{N},
$$

where $\xi_{N}$ is the step size scaled by tick $N$, so that $\xi_{N} \rightarrow 0$ as $N \rightarrow \infty$. The columns of the matrix, $\mathbf{U}_{N}$, are the sequential updates for the $q$ largest eigenvectors, i.e., $\mathbf{U}_{N} \mathbf{U}_{N}^{T}$ is the projection matrix we use in the sequential version (3.1). As pointed out by Oja and Karhunen (1985) and Oja (1992), $\Psi$ is in fact unknown. A natural approach is to consider the adaptive estimator of Equation (3.10) as an estimator for $\Psi$. As a new vector $\mathbf{x}_{N+1}$ arrives, the update is

$$
\begin{aligned}
& \tilde{\mathbf{U}}_{N+1}=\mathbf{U}_{N}+\xi_{N}\left(\mathbf{x}_{N+1}-\boldsymbol{\mu}_{N+1}\right)\left(\mathbf{x}_{N+1}-\boldsymbol{\mu}_{N+1}\right)^{T} \mathbf{U}_{N} \\
& \mathbf{U}_{N+1}=\Pi\left(\tilde{\mathbf{U}}_{N+1}\right),
\end{aligned}
$$

where $\Pi(\cdot)$ denotes an orthonormalization operator. Orthonormalization can be achieved, for instance, using a Gram-Schmidt (GS) procedure. In Oja (1992), a sequential version of GS was proposed by combining Equations (3.15)-(3.16) (detailed in Equations (8.6)-(8.8) of Appendix 8.2). This operator is necessary to guarantee orthonormality as is required by PCA. The proposal included Robbins-Monro conditions on the sequence of $\xi_{N}$ to ensure convergence (Oja, 1992; Oja and Karhunen, 1985), i.e., $\sum_{N \geq 1} \xi_{N}^{2}<\infty$ 
and $\sum_{N \geq 1} \xi_{N}=\infty$. This condition is clearly satisfied in the case when $\xi_{N}$ is scaled by tick $N$. This procedure, while theoretically well justified for i.i.d. data, requires modification to match the requirements of streaming data analysis. In particular, we do not scale the sequence of $\xi_{N}$ to decrease with time as is required to satisfy the Robbins-Monro conditions and conduct an empirical study about it on Section 3.6. Future data may not be generated by the same process as current data and hence it is undesirable to suppress the learning capabilities of the estimator. Bearing this in mind, we modified the results in Oja (1992), using the adaptive estimators $\boldsymbol{\mu}_{N, \vec{\lambda}}$ and $\boldsymbol{\Sigma}_{N, \vec{\lambda}}$, to update the $j$-th column of the matrix $\mathbf{U}_{N+1}$ as

$$
\begin{aligned}
& \mathbf{u}_{j, N+1}= \mathbf{u}_{j, N}+\xi \phi_{j, N} \\
& {\left[\left(\mathbf{x}_{N+1}-\boldsymbol{\mu}_{N+1, \vec{\lambda}}\right)-\phi_{j, N} \mathbf{u}_{j, N}-2 \sum_{i=1}^{j-1} \phi_{i, N} \mathbf{u}_{i, N}\right] } \\
& \phi_{j, N}=\left(\mathbf{x}_{N+1}-\boldsymbol{\mu}_{N+1, \vec{\lambda}}\right)^{T} \mathbf{u}_{j, N} \\
& \gamma_{j, N+1}=\gamma_{j, N}+\xi\left(\phi_{j, N}^{2}-\gamma_{j, N}\right),
\end{aligned}
$$

denoting $\gamma_{j, N}$ as the $j$-th largest eigenvalue at time $N$ and $\phi_{j, N}$ is an auxiliary quantity. The subscript of $\xi$ was removed to denote that this is a fixed value henceforth. Note however that the sequential update (3.17) is an approximation of the orthonormalization operator $\Pi(\cdot)$ of Equation (3.16). As a drawback, the approximation does not guarantee orthogonality and in practice it might even be loss due to round-off errors. Later, in Section 3.5 we explore some properties when the approximation method is adopted.

3.4. Computational implementation. In this section we present two implementations of streaming PCA, which we refer to as Multivariate Adaptive Forgetting Factor (MAFF) PCA, which uses adaptive estimation, and Multivariate Fixed Forgetting Factor (MFFF) PCA. The main difference between the two methods is that MAFF uses time-varying forgetting factors, tuned as in Equation (3.11). Later we prefer MAFF since it alleviates the need to select, and rely on, a single forgetting factor in perpetuity.

The step by step implementation of MFFF is implemented in Algorithm 1 while MAFF is detailed in Algorithm 2. Note that both algorithms require a burn-in period, based on $\mathcal{B}$ consecutive measurements. Both algorithms thus feature a burn-in phase, followed by adaptive and sequential updating. Note also that both algorithms include a sequential anomaly detection stage, described in Section 4.

In Algorithm 1, first select the value for the FF and step size $\xi \in \mathbb{R}^{+}$. There is no principled method to select the FF in advance. Then initial 
values for the recursive estimators are set as $\mathbf{m}_{1, \lambda}=\mathbf{x}_{1}, w_{1, \lambda}=1, \Sigma_{1}=I_{d}$. Note that the updates of $\mathbf{m}_{N+1, \lambda}, w_{N+1, \lambda}, \boldsymbol{\mu}_{N+1, \lambda}$ and $\Sigma_{N+1, \lambda}$ are the same as Equations (3.6), (3.7), (3.9), (3.10). The only difference is that $\lambda_{N}=\lambda$. Other input parameters, such as $\xi$, will be determined by simulation studies based on properties of the real data.

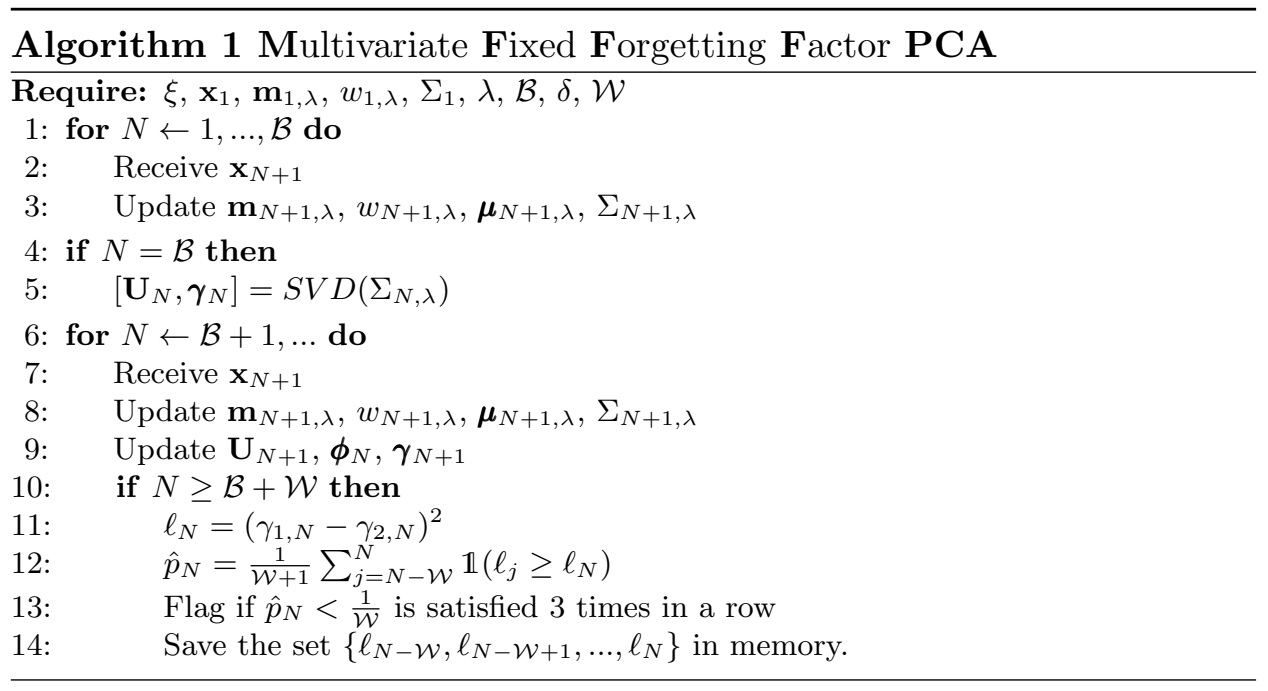

For the implementation of MAFF, Algorithm 2, first select the values for both step sizes $\xi \in \mathbb{R}^{+}$and $\eta \in \mathbb{R}^{+}$. Then initial values for the recursive estimators are set as $\mathbf{m}_{1, \vec{\lambda}}=\mathbf{x}_{1}, \Delta_{1, \vec{\lambda}}=\mathbf{x}_{1}, w_{1, \vec{\lambda}}=1, \Omega_{1, \vec{\lambda}}=1$, $\Sigma_{1}=I_{d}, \lambda_{1}=1, \lambda_{\min }=0.6$. After updating $\lambda_{N}$, the equation $\lambda_{N+1}=$ $\max \left\{\min \left\{\lambda_{N+1}, 1\right\}, \lambda_{\min }\right\}$ in Line 4 , is to guarantee that $\lambda_{N} \in\left(\lambda_{\min }, 1\right)$.

The first $\mathcal{B}$ ticks, corresponding to a burn-in, are used to estimate $\mathbf{U}_{N}$ and $\boldsymbol{\gamma}_{N+1}$ using SVD (Lines 1-5 in Algorithm 1 and Lines 1-6 in Algorithm 2 ). After this burn-in the estimation of the eigendecomposition is sequential and adaptive. Note that the vectors $\boldsymbol{\phi}_{N}$ and $\boldsymbol{\gamma}_{N}$ in Lines 9 (Algorithm 1) and 11 (Algorithm 2) are updated after the burn-in using Equation (3.18) and Equation (3.19), respectively.

This instrumented infrastructure application involves data measured at high frequency, and so it is reasonable to set the burn-in period of both algorithms $\mathcal{B}$ to 500 ticks. Post burn-in, anomaly detection is performed by retaining a set of $\mathcal{W}$ derived values, see Lines 10-14 (Algorithm 1) and 12-16 (Algorithm 2). This set is used to calculate a $p$-value which provides the basis of the anomaly detector.

3.5. Orthogonality and computational times. We now evaluate the approximation presented in Equations (3.17)-(3.19) in terms of orthogonality 


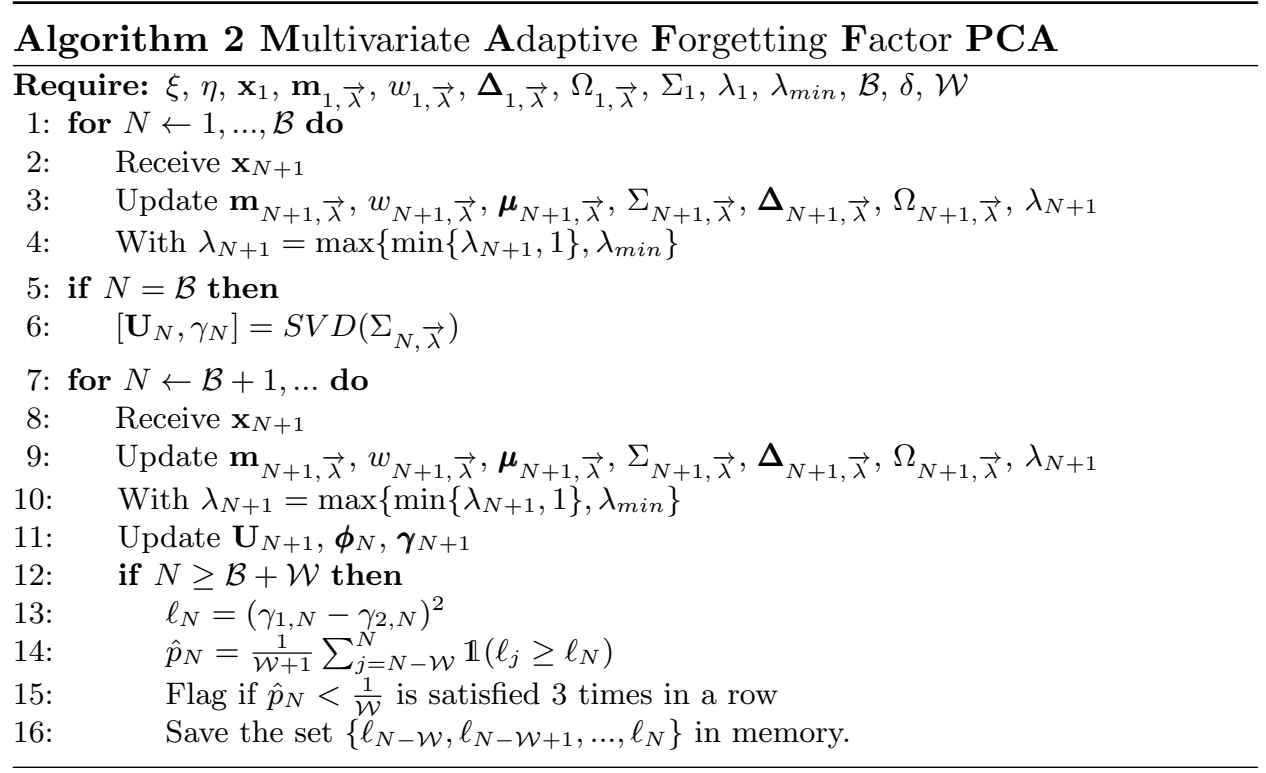

loss and computational times when comparing it to the exact GS procedure. A simulation experiment was conducted generating 100 replicates and length $N=40500$ ticks of an i.i.d. data generation process (see 8.4.1 for more details) and varying the number of eigenvalues and eigenvectors to update, $q=\{2,5,20,50,80\}$. MAFF parameters were set as discussed in Section 6 . The loss of orthogonality is evaluated taking the average of

$$
\frac{1}{N} \sum_{j=1}^{N}\left\|I_{q}-\mathbf{U}_{j}^{T} \mathbf{U}_{j}\right\|_{F}
$$

over 100 Monte Carlo replicates, where, using the same notation of Equation (3.1), $\|\cdot\|_{F}$ denotes the Frobenius norm and $I_{q}$ denotes the $q$ dimensional identity matrix. Such metric was adopted in a previous work (Giraud et al., 2005) to evaluate the loss of orthogonality when using computational variants of GS. Results are reported on the left plot of Figure 7. As expected the basis remains orthogonal when using exact GS. A striking feature is that there is almost zero loss considering $q=2$ eigenvectors in the approximated version.

The average computational time was also evaluated in seconds on logarithm scale. While the exact computational time of GS takes $O\left(q^{2} d\right)$, where $d=80$ in our application, the approximated version takes $O(q d)$ (Cardot and Degras, 2018). This corroborates with the findings presented in the top right and bottom plots of Figure 7, where the computed times are approxi- 
mately linear in $q$. Note the non-linear horizontal axes in the top right plot in Figure 7.

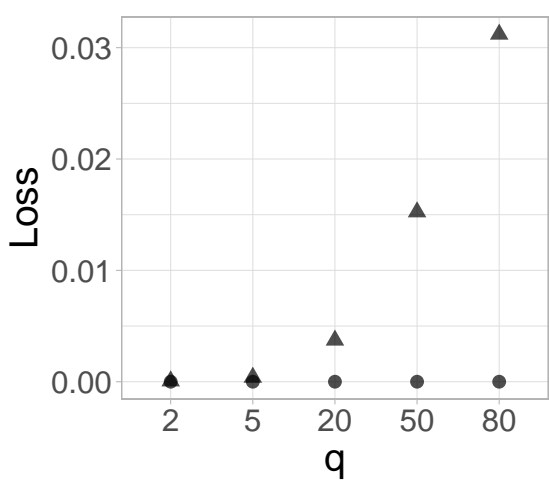

- Exact $\Delta$ Approximated

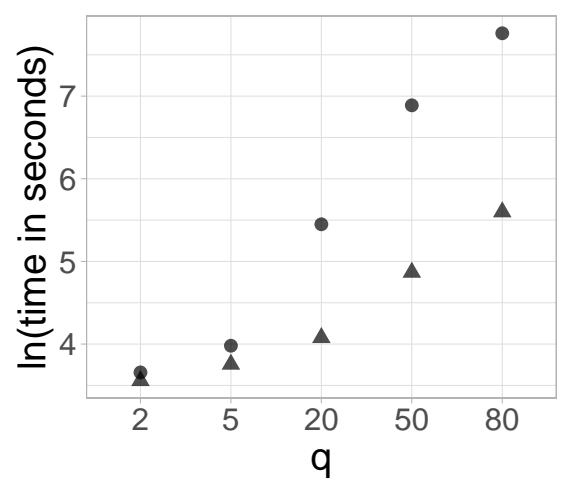

- Exact $\Delta$ Approximated

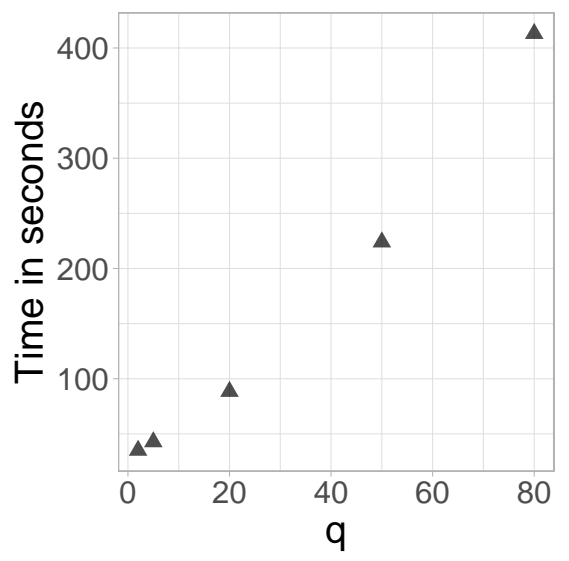

Fig 7: Figures elucidating the loss of orthogonality and computational times considering the update of the first $q=\{2,5,20,50,80\}$ eigenvalues and eigenvectors, also with the approximation and exact Gram-schmidt operator. Note that the horizontal axes in both plots at the top are non-linear.

3.6. Algorithm stability. As a novel methodological contribution, the proposed streaming algorithm in this paper is relaxing the Robins-Monroe conditions (Oja and Karhunen, 1985; Oja, 1992). In this section we demonstrate that relaxing the Robins-Monroe's condition leads to stability in terms of parameter estimation.

Figure 8 display the results of Monte Carlo experiments involving i.i.d. data for which both the first and the second eigenvalue equal 1 (True). The 
top plot in Figure 8 shows the Monte Carlo average (100 replicates) of the estimated first eigenvalue over the course of the experiment, while the bottom plot shows the corresponding second eigenvalue. Both plots in Figure 8 refers to MAFF with (RB) and without (WRB) the Robbins-Monroe condition (Equations (3.17)-(3.19)). MAFF parameters were set as mentioned in Section 6. Interestingly the MAFF estimate appears unbiased and reasonably behaved, which is not true for the estimate using the Robbins-Monroe condition. The results of this i.i.d. simulation provide empirical evidence that our MAFF procedure (that relaxes the Robins-Monroe condition) does not produce unstable parameter estimates.
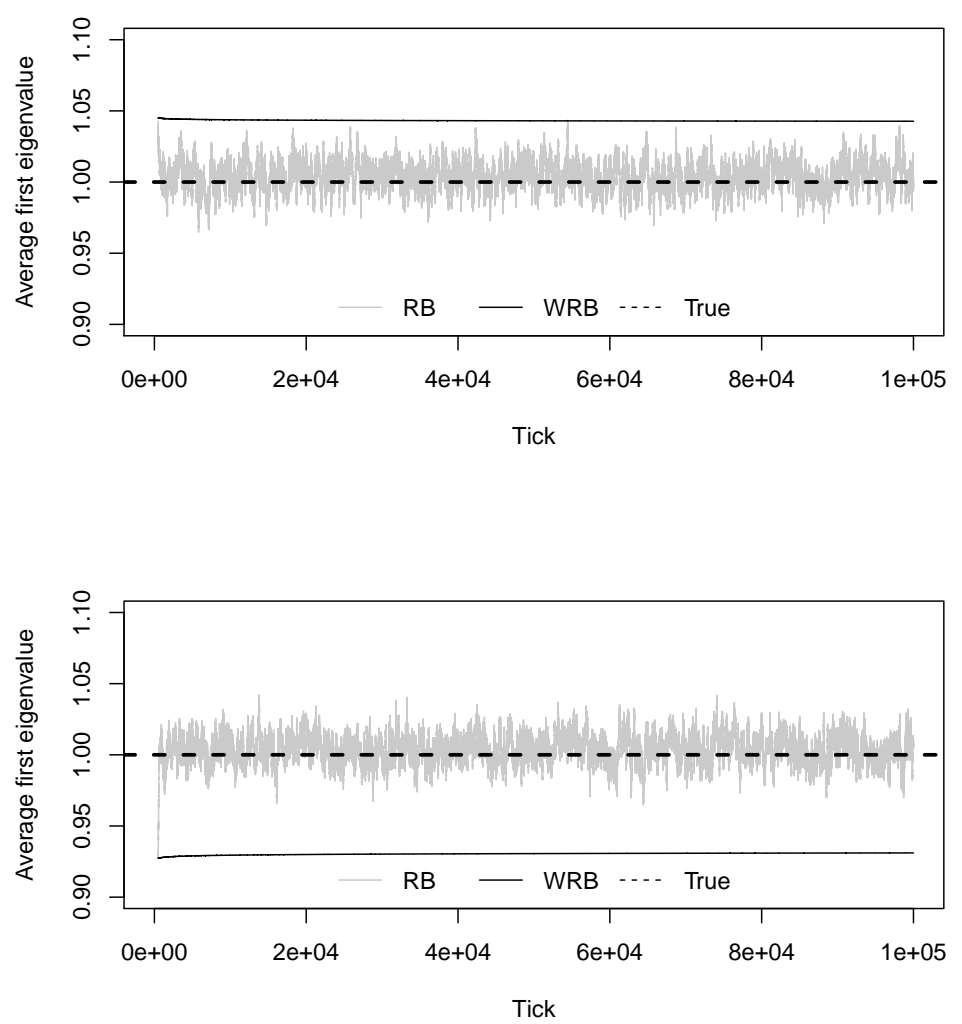

Fig 8: Average of Monte Carlo replicates of first and second eigenvalues. Both plots refers to MAFF without using the Robbins-Monroe condition. A horizontal dashed line was introduced in both plots to illustrate true values of both eigenvalues. 
4. Anomaly detection. Given the estimation methodology introduced in the previous section, we now develop an anomaly detection mechanism. In general, inference for the parameters of an eigendecomposition is difficult, and there are few results on the distribution of such estimators (Johnstone, 2001; Nadler, 2011). One can reason about tracking the largest eigenvalue in PCA (Johnstone, 2001), or even the ratio of the largest eigenvalue to the sum of all eigenvalues (Nadler, 2011), using the Tracy-Widom distribution. However, these results require assumptions based on asymptotic theory which are not valid for streaming data. A different approach is required.

To overcome these issues and avoid making distributional assumptions, we adapt Conformal Prediction (CP) to propose a streaming PCA anomaly detector. CP was developed by the machine learning community (Vovk et al., 2005, 2009; Vovk, 2013; Burnaev and Vovk, 2014; Balasubramanian et al., 2014) and is now receiving substantial attention from the statistics community (Lei et al., 2013, 2015, 2018; Lei and Wasserman, 2014; Chernozhukov et al., 2018). CP methods require storage of $\mathcal{W}$ derived quantities. These derived quantities are called non-conformity measures, which makes reference to a distance metric specified by the user. Selecting an appropriate measure remains an open problem in the literature (Vovk et al., 2005). We propose a particular measure that is designed for this instrumented infrastructure application, motivated by the behaviour depicted in Figures 5c and 5d.

4.1. Non-conformity measure. Given the multivariate data stream $\mathbf{x}_{N}$ and the eigenvalues derived from it, $\gamma_{N}$, we seek to construct a non-conformity measure with which to perform inference.

To monitor for anomalies, the sequence of eigenvalues is converted into the stream of distances

$$
\left\langle\ell_{2}, \ell_{3}, \ldots, \ell_{N-1}, \ell_{N}, \ldots\right\rangle, \quad \ell_{N} \equiv \mathrm{D}\left(\gamma_{1, N}, \gamma_{2, N}\right)
$$

using any valid distance metric $\mathrm{D}(\cdot, \cdot)$. Inspection of extensive bridge data shows that during at-rest periods the eigendecomposition is consistent with that depicted in Figures 5a and 5c. In contrast during train passage events, there is a significant difference between the two first eigenvalues, which are approximately equal during periods of rest. Hence Equation (4.1) restricts attention to the first two eigenvalues. This motivates the use of a non-conformity measure

$$
\ell_{N}=\left(\gamma_{1, N}-\gamma_{2, N}\right)^{2} .
$$

Of course, other measures are possible, but as noted above there is no principled way to select a measure. 
Following $\mathrm{CP}$ we sequentially estimate a $p$-value as

$$
\hat{p}_{N}=\frac{1}{\mathcal{W}+1} \sum_{j=N-\mathcal{W}}^{N} \mathbb{1}\left(\ell_{j} \geq \ell_{N}\right),
$$

where $\mathbb{1}(\cdot)$ is the indicator function. Generally $\mathrm{CP}$ would allow the set of derived quantities, $\mathcal{W}$, to increase with data arrival. This is not suitable for streaming data contexts because both the computational and memory demand would increases over time (Domingos and Hulten, 2003). Instead we restrict this set to a sliding window of fixed size, adapting ideas from Laxhammar and Falkman (2015). By construction $\hat{p}_{N} \in\left[\frac{1}{\mathcal{W}+1}, 1\right]$.

Equation (4.3) defines the $p$-value, $\hat{p}_{N}$, which forms the basis of our anomaly detection system. As is often the case in practical sequential analysis, it is useful to incorporate a "run rule" (Champ and Woodall, 1987), which both reduces false positives and provides some resistance to outliers. We will adopt a run rule in which an anomaly is flagged at tick $j$ if $\hat{p}_{j}$, $\hat{p}_{j-1}$ and $\hat{p}_{j-2}$ are all less than $\alpha=\frac{1}{\mathcal{W}}$. The latter implies that at least one anomaly will be detected, on average, after observing a set of $\mathcal{W}$ measurements. Theoretically, setting $\mathcal{W}$ large is desirable. Practically however, a balance must be struck between memory and computing constraints. For the bridge data, experimentation suggests that $\mathcal{W}=10000$ (equivalent to 40 seconds), provides good results.

5. Simulation. In this section, the performance of these streaming PCA methods, MFFF (Algorithm 1) and MAFF (Algoritm 2), are evaluated. The scaled version of MFFF (as discussed in Section 3.3), in which the Robins-Monroe conditions are satisfied, does not perform well in simulations and is not considered in detail. This latter approach, which makes little sense in a streaming context, is denoted as MFFFS.

These three methods are compared based on Estimation Accuracy and Detection Performance. The first is intended to evaluate how well the algorithm tracks properties of a time-varying eigendecomposition. The second measures the effectiveness of the method proposed in Section 4, at detecting anomalies. It is well known that such computational methods are sensitive to the choice of input parameters. Thus, these simulations are also used to determine input parameters for the bridge data analysis of Section 6. Details of the search grid for input parameters are presented in Appendix 8.3.

We simulate data, $X \in \mathbb{R}^{N \times d}$, that reproduces important characteristics reported in Section 2, with $d=80$ for consistency with the bridge data and $N=40500$ ticks. The key features to be replicated are the annular structure observed during at-rest periods and its collapse during train passage events. 
A detailed description of the data generation processes is given in Appendix 8.4.

Considering Estimation Accuracy, recall that $\gamma_{j}$ is a vector of the 2 leading eigenvalues estimated by a streaming PCA procedure. In the simulated setting the corresponding true eigenvalues are denoted as $\boldsymbol{\Gamma}_{j}$. Accuracy is measured using

$$
\frac{1}{N} \sum_{j=1}^{N}\left\|\boldsymbol{\Gamma}_{j}-\gamma_{j}\right\| .
$$

We will report the average of this error measure, $\mathcal{E}$, over 100 Monte Carlo replicates considering i.i.d. data (data generation described in Appendix 8.4.1).

To identify whether a flagged change corresponds to a real anomaly in simulation studies we will use a window around the real anomaly. Recall we use a run rule which flags the anomaly as the first tick in the run. Similar to Kim et al. (2009), if a flag is given in a window of $\delta=125$ measurements following a true anomaly, the detection is deemed a correct detection (CD). A flag outside this window is deemed a false detection (FD). The tolerance period, $\delta$, is equivalent to half a second for these data. Average number of FD and the CD rates are calculated over 100 replicates of simulated train passage event data (see Appendix 8.4.2).

5.1. Simulation results and control parameter selection. We seek to compare MAFF and MFFF according to various measures of performance. Additionally we use the results of the simulation study to identify control parameters for Section 6.

\begin{tabular}{|c|c|c|c|c|c|c|c|c|c|}
\hline$\eta$ & $\xi$ & $\mathcal{E}$ & CD & $\mathrm{FD}$ & & & & & \\
\hline 0.1 & 0.01 & 0.17 & 0.70 & 3.40 & $\lambda$ & $\xi$ & $\mathcal{E}$ & CD & $\mathrm{FD}$ \\
\hline 0.01 & 0.01 & 0.10 & 0.61 & 3.66 & $\lambda$ & 5 & $c$ & 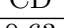 & TD \\
\hline 0.001 & 0.01 & 0.09 & 0.75 & 3.45 & 0.85 & 0.01 & 0.22 & 0.63 & 3.80 \\
\hline $1 \mathrm{e}-04$ & 0.01 & 0.08 & 0.76 & 2.99 & 0.9 & 0.01 & 0.15 & 0.67 & 3.60 \\
\hline $1 \mathrm{e}-05$ & 0.01 & 0.08 & 0.70 & 3.76 & 0.95 & 0.01 & 0.10 & 0.76 & 3.37 \\
\hline $1 \mathrm{e}-06$ & 0.01 & 0.08 & 0.77 & 3.58 & 0.99 & 0.01 & 0.08 & 0.79 & 3.35 \\
\hline $1 \mathrm{e}-07$ & 0.01 & 0.08 & 0.73 & 3.33 & & & & & \\
\hline
\end{tabular}

Table 1: Average results over 100 Monte Carlo replicates, where the first two columns are control parameters of each method, $\mathcal{E}$ is the average error. CD is expressed as a rate and FD is the average number of points. Left: results for MAFF. Right: results for MFFF.

Table 1 reports the results of the performance measures for MAFF and MFFF, left and right, respectively. The first two columns of each table re- 
fer to input parameters for the respective methods. Note, these tables have been reduced to report only configurations of input parameters which have $\mathrm{CD}>0.5$ and $\mathrm{FD}<4$. The full tables are available in Appendix 8.5. In simulations, not presented here, we compared the performance of square error and absolute error as non-conformity measures in (4.2). Results were similar for both measures. The complete results demonstrate that good performance requires very specific choice of parameters.

The results in Tables 1 embody two scenarios. First, i.i.d. data which is included to address estimation issues alone. The column denoted $\mathcal{E}$ reports the average estimation accuracy, Equation (5.1). Interestingly, the parameter $\xi$, which has the same role in both methods, the value 0.01 is very frequently selected as a good choice.

In terms of detection performance, simulated train passage event data was used to assess detection performance, as reported in the CD and FD column of Table 1 . These tables indicate broadly the same performance over the reported input parameters. Specifically, a CD rate of around 0.7 and FD around 3.5. Without using the run-rule, with $\mathcal{W}=10000$ an average of four false detections is expected.

As always, different performance measures demonstrate different characteristics for fixed choices of parameters, and hence our selection will seek to balance estimation accuracy and detection performance. This balance is biased in favour of detection performance which itself is characterised by two measures, CD and FD.

Given the simulation results, we use MAFF for the bridge data with parameters $\eta=1 e-6, \xi=0.01$. We favour MAFF over MFFF, because fixing $\lambda$ in perpetuity seems an overcommitment of knowledge.

Finally, note that MFFFS behaves in a predictable and practically useless manner. Specifically, MFFFS is shown by simulation (see Table 4 in the Appendix 8.5) to be incapable of adaptively revising its estimates following the train passage event. This is unsurprising since the scaling of MFFFS renders adaptive learning impossible after sufficient data have been observed.

6. Bridge Data. In this section we demonstrate the result of our MAFF approach for the bridge data described in Section 2. Input parameters selected in the previous section were used throughout this section.

As noted earlier, the existence of the latent annular structure, illustrated in Figure 5a, has rarely been documented, a notable exception being Novembre and Stephens (2008). This structure is persistent when evaluated over sliding windows during periods of rest. Notably when using MAFF, this structure is preserved, see Figure 9. 


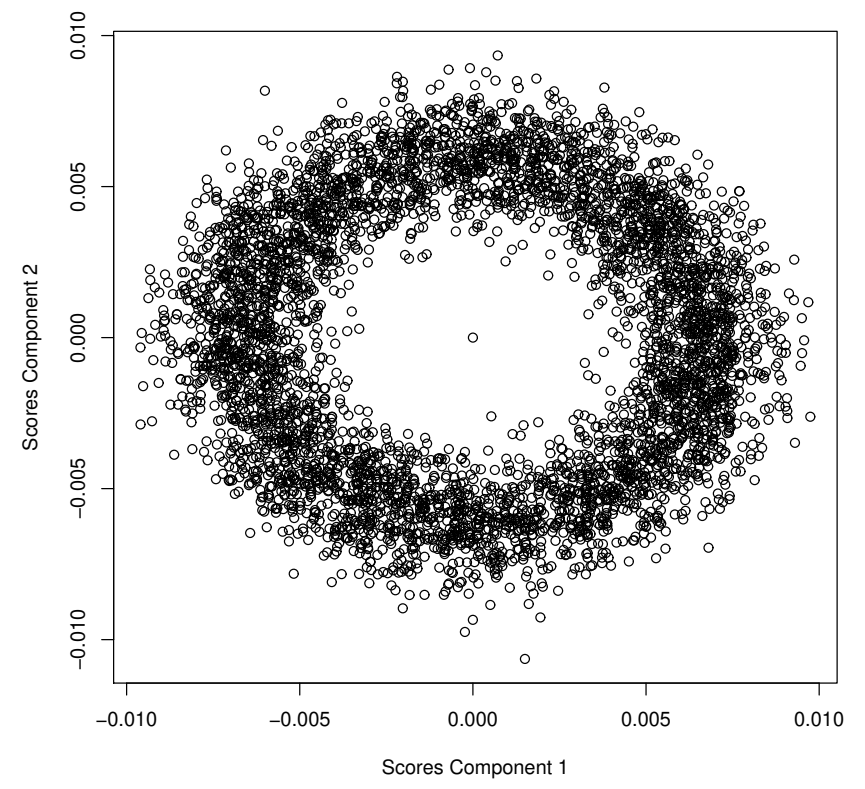

Fig 9: Online annulus calculated considering 5000 data points, sequentially centred with $\boldsymbol{\mu}_{N+1, \vec{\lambda}}$, and a selected $\mathbf{U}_{N}$ during an at-rest period.

The MAFF method also reveals a previously unknown feature of this type of distributed sensor system. Figure 10 shows the maximum eigenvalue computed by MAFF over a long period. There were four train passage events in this period. The striking feature of this figure does not relate to train passage events but rather is the obvious periodicity. The periodicity is very close to 5 minutes and 40 seconds. Neither we, nor engineering colleagues, are able to explain this beyond attributing it to spatio-temporal proprieties of the sensor system. This previously unknown and unanticipated latent time series structure is one of a number of dynamic phenomena we have discovered. These latent structures would certainly be amenable to modelling with time series approaches, and this will be the subject of a forthcoming treatment. Our purpose with this demonstration is to show that the modelling approach reveals, as a by-product, a deeper aspect of the data generating process.

Turning now to anomaly detection, recall that the data set has four train passage events, which were manually identified. Each frame of Figure 11 shows $\hat{p}_{N}$ zoomed-in around the train passage events. MAFF misses only one of these four events. Excluding the four train event periods the anomaly 


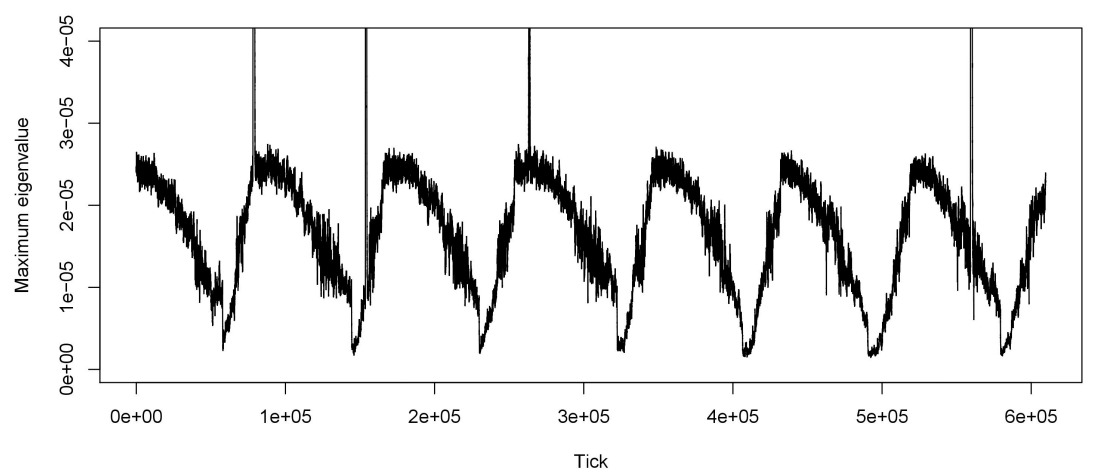

Fig 10: Maximum eigenvalue sequentially estimated by MAFF over 611108 data points (approximately 40 minutes and 44 seconds). The four spikes corresponds to train passage events. The maximum value on the vertical axis was selected so the cyclic feature is more evident.

detector signalled $0.0262 \%, 157$ ticks of 598422, as false positives.

Finally, considering computational burden, we need to address memory and efficiency issues. MAFF has constant memory and compute demand per tick, as required by streaming applications. In the example just given, the entire data set consists of 611108 measurements which is equivalent to 40 minutes and 44 seconds on a $250 \mathrm{~Hz}$ frequency sample. The code developed in $\mathrm{R}$, not optimized, required 8 minutes and 49 seconds to process the data. Thus the procedure is capable of processing such data in real time. 

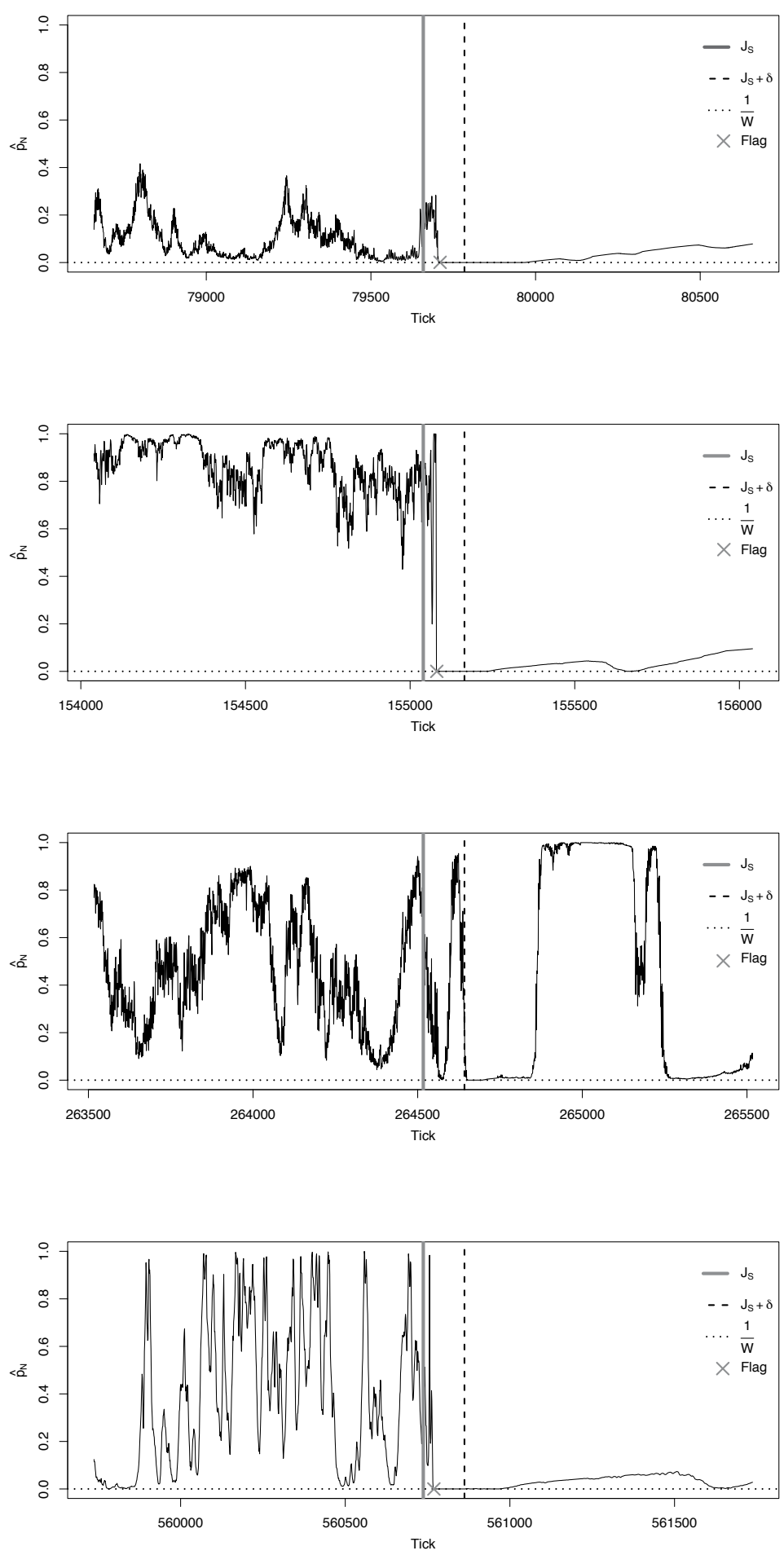

Fig 11: Conformal $p$-values, $\hat{p}_{N}$, for flagged periods. Solid vertical lines denote the tick in which the train passage event started. The interval between the solid vertical lines and dashed vertical lines denote the tolerance period $\delta$. Horizontal dotted lines indicate $\alpha=\frac{1}{\mathcal{W}}$. The red cross indicates the first tick for which a flag occurred, based on the run rule. 
7. Conclusion. We have considered data analysis challenges arising from instrumented infrastructure. Specifically, we have developed a novel streaming methodology for multivariate anomaly detection over a spatiotemporal object. MAFF has been extended using an adaptive forgetting factor and derived quantities from the method are calibrated using Conformal Prediction in a fixed window. Given appropriate control parameters, and a run-rule, the algorithm provides both effective tracking performance and accurate detection capability.

Deployed against real bridge data, the method has an acceptable detection performance for train passage events. Notably the method reveals a long term cyclic dependence structure that has not been previously reported. Forthcoming work will investigate stochastic mechanisms to model the annular structure exploit in this paper.

Acknowledgments. The authors would like to acknowledge the Cambridge Centre for Smart Infrastructure and Construction (CSIC), The Laing O'Rourke Centre at Cambridge and the Staffordshire Alliance (Network Rail, Volker Rail, Atkins and Laing O'Rourke) for providing the dataset used in the paper. The third author would like to acknowledge support from the The Alan Turing Institute under the EPSRC grant EP/N510129/1 and the Turing-Lloyd's Register Foundation Programme for Data-Centric Engineering. The work of Henrique Helfer Hoeltgebaum was fully supported by the National Council for Research and Development, CNPq, Ministry of Science and Technology, Brazil. We are grateful to Heather Battey and Dean Bodenham for valuable comments on this manuscript. Also we are grateful to the editor and two anonymous reviewers for their insightful comments.

\section{Appendix.}

8.1. Proof. Equivalence between Equation (3.8) and Equation (3.9) considering the univariate case. 


$$
\begin{aligned}
\bar{y}_{N, \lambda} & =\frac{m_{N, \lambda}}{w_{N, \lambda}} \\
\bar{y}_{N, \lambda} & =\frac{\lambda m_{N-1, \lambda}+y_{N}}{w_{N, \lambda}} \\
\bar{y}_{N, \lambda} & =\frac{\lambda w_{N-1, \lambda} \bar{y}_{N-1, \lambda}}{w_{N, \lambda}}+\frac{y_{N}}{w_{N}} \\
\bar{y}_{N, \lambda} & =\frac{\left[w_{N, \lambda}-1\right] \bar{y}_{N-1, \lambda}}{w_{N, \lambda}}+\frac{y_{N}}{w_{N}} \\
\bar{y}_{N, \lambda} & =\left(1-\frac{1}{w_{N, \lambda}}\right) \bar{y}_{N-1, \lambda}+\frac{y_{N}}{w_{N}}
\end{aligned}
$$

8.2. Stochastic Gradient Ascent. In order to update the $j$-th column of the matrix $\mathbf{U}_{N+1}$ under orthonormality conditions, Oja (1992) proposed the following

$$
\begin{aligned}
& \mathbf{u}_{j, N+1}= \mathbf{u}_{j, N}+\xi_{N} \phi_{j, N} \\
& {\left[\left(\mathbf{x}_{N+1}-\boldsymbol{\mu}_{N+1}\right)-\phi_{j, N} \mathbf{u}_{j, N}-2 \sum_{i=1}^{j-1} \phi_{i, N} \mathbf{u}_{i, N}\right] } \\
& \phi_{j, N}=\left(\mathbf{x}_{N+1}-\boldsymbol{\mu}_{N+1}\right)^{T} \mathbf{u}_{j, N} \\
& \gamma_{j, N+1}=\gamma_{j, N}+\xi_{N}\left(\phi_{j, N}^{2}-\gamma_{j, N}\right),
\end{aligned}
$$

where $\xi_{N}=\frac{\xi}{N}$.

8.3. Input parameters. The lack of theoretical background on the optimal choice of SGD step size (Goodfellow et al., 2016) motivates the exploration of a grid of values for the proposed methods. The adopted grids are

$$
\xi \in\left\{10^{-1}, 10^{-2}, 10^{-3}, 10^{-4}, 10^{-5}, 10^{-6}\right\},
$$

for the eigendecomposition step,

$$
\eta \in\left\{10^{-1}, 10^{-2}, 10^{-3}, 10^{-4}, 10^{-5}, 10^{-6}\right\},
$$

for the adaptive forgetting factor step and

$$
\lambda \in\{0.6,0.75,0.8,0.85,0.9,0.95,0.99\}
$$

for the FF. This simulation study explores all possible combinations of $\xi, \eta$ and $\lambda$. 
8.4. Data generation process. For both data generation processes, calculate the sample covariance matrix $\Sigma$ as

$$
N \Sigma=X^{T} X=\mathbf{U} \Lambda \mathbf{U}^{T}
$$

where $\Lambda$ denotes a diagonal matrix composed of the eigenvalues $\gamma_{1} \geq \gamma_{2} \geq$ $\ldots \geq \gamma_{80}$.

8.4.1. IID data. In order to generate the i.i.d. data set, with only resting periods, one should adopt the following steps.

1. Generate one random orthonormal matrix, $\Upsilon \in \mathbb{R}^{80 \times 80}$ using $\mathrm{QR}$ decomposition Mezzadri (2006) or Householder projections Stewart (1980);

2. Generate one diagonal matrix $A \in \mathbb{R}^{80 \times 80}$ with elements $A_{[1,1]}=1$, $A_{[2,2]}=0.01, A_{[j, j]} \sim \operatorname{Unif}\left[-10^{-6}, 10^{-6}\right], \forall j=3, \ldots 80$. Note that the notation $\psi_{[j, j]}$ makes reference to the element in the $j$-th row, $j$-th column of the $\psi$ matrix.

3. Fixing 40500 as the sample size, generates the stream $X \in \mathbb{R}^{40500 \times 80} \sim$ $\mathcal{N}\left(\boldsymbol{\mu}, \Upsilon^{T} A \Upsilon\right)$ where $\mathcal{N}(\boldsymbol{\mu}, \Sigma)$ denotes a multivariate Gaussian distribution, with mean vector $\boldsymbol{\mu}=[1,2, \ldots, 80] \in \mathbb{R}^{80}$ and covariance matrix $\Sigma$. Such mean vector was proposed since the sensors are aligned in an increasing sequence equally spaced.

8.4.2. Train passage event data. To generate data considering a rest period, followed by a train passage event and followed by another rest period, we adopted the following steps.

1. Generate two random orthonormal matrixes, $\Upsilon \in \mathbb{R}^{80 \times 80}$ and $\Xi \in$ $\mathbb{R}^{80 \times 80}$ using QR decomposition Mezzadri (2006) or Householder projections Stewart (1980);

2. Generate two diagonal matrixes $A \in \mathbb{R}^{80 \times 80}$ and $B \in \mathbb{R}^{80 \times 80}$ with elements $A_{[1,1]}=1, A_{[2,2]}=0.01, A_{[j, j]} \sim \operatorname{Unif}\left[-10^{-6}, 10^{-6}\right], \forall j=$ $3, \ldots 80$ and $B_{[1,1]}=5 B_{[j, j]} \sim \operatorname{Unif}\left[-10^{-6}, 10^{-6}\right], \forall j=2, \ldots 80$. Note that the notation $\psi_{[j, j]}$ makes reference to the element in the $j$-th row, $j$-th column of the $\psi$ matrix.

3. Fixing 40500 as the sample size, generates the stream $X \in \mathbb{R}^{40500 \times 80}$ as

$$
X_{N}= \begin{cases}\mathcal{N}\left(\boldsymbol{\mu}, \Upsilon^{T} A \Upsilon\right), & N \leq 20000, \\ \mathcal{N}\left(\boldsymbol{\mu}, \Xi^{T} B \Xi\right), & 20001 \leq N \leq 20500, \\ \mathcal{N}\left(\boldsymbol{\mu}, \Upsilon^{T} A \Upsilon\right), & 20501 \leq N \leq 40500\end{cases}
$$


where $\mathcal{N}(\boldsymbol{\mu}, \Sigma)$ denotes a multivariate Gaussian distribution, with mean vector $\boldsymbol{\mu}=[1,2, \ldots, 80] \in \mathbb{R}^{80}$ and covariance matrix $\Sigma$. Such mean vector was proposed since the sensors are align in an increasing sequence equally spaced.

8.5. Simulation results. In this section, we report the results of Section 5 for the whole grid of input parameters. The average error metric of Equation (5.1), considering i.i.d. context and also the CD and FD performances on the train passage event scenario for MAFF (Table 2), MFFF (Table 3) and MFFFS (Table 4). 


\begin{tabular}{ccccr}
\hline$\eta$ & $\xi$ & $\mathcal{E}$ & $\mathrm{CD}$ & \multicolumn{1}{c}{$\mathrm{FD}$} \\
\hline 0.1 & 0.1 & 0.27 & 0.41 & 68.01 \\
0.1 & 0.01 & 0.17 & 0.70 & 3.40 \\
0.1 & 0.001 & 0.16 & 0.00 & 2.32 \\
0.1 & $1 \mathrm{e}-04$ & 0.17 & 0.00 & 20.93 \\
0.1 & $1 \mathrm{e}-05$ & 0.23 & 0.00 & 98.77 \\
0.1 & $1 \mathrm{e}-06$ & 0.29 & 0.01 & 213.61 \\
0.1 & $1 \mathrm{e}-07$ & 0.28 & 0.00 & 126.96 \\
0.01 & 0.1 & 0.26 & 0.34 & 203.80 \\
0.01 & 0.01 & 0.10 & 0.61 & 3.66 \\
0.01 & 0.001 & 0.08 & 0.00 & 2.62 \\
0.01 & $1 \mathrm{e}-04$ & 0.10 & 0.00 & 14.32 \\
0.01 & $1 \mathrm{e}-05$ & 0.14 & 0.00 & 85.11 \\
0.01 & $1 \mathrm{e}-06$ & 0.15 & 0.00 & 97.74 \\
0.01 & $1 \mathrm{e}-07$ & 0.19 & 0.00 & 92.91 \\
0.001 & 0.1 & 0.26 & 0.35 & 203.77 \\
0.001 & 0.01 & 0.09 & 0.75 & 3.45 \\
0.001 & 0.001 & 0.04 & 0.00 & 2.92 \\
0.001 & $1 \mathrm{e}-04$ & 0.05 & 0.00 & 11.04 \\
0.001 & $1 \mathrm{e}-05$ & 0.10 & 0.00 & 32.81 \\
0.001 & $1 \mathrm{e}-06$ & 0.10 & 0.00 & 52.15 \\
0.001 & $1 \mathrm{e}-07$ & 0.11 & 0.00 & 44.40 \\
$1 \mathrm{e}-04$ & 0.1 & 0.26 & 0.40 & 271.73 \\
$1 \mathrm{e}-04$ & 0.01 & 0.08 & 0.76 & 2.99 \\
$1 \mathrm{e}-04$ & 0.001 & 0.03 & 0.00 & 3.12 \\
$1 \mathrm{e}-04$ & $1 \mathrm{e}-04$ & 0.02 & 0.00 & 9.11 \\
$1 \mathrm{e}-04$ & $1 \mathrm{e}-05$ & 0.05 & 0.00 & 20.02 \\
$1 \mathrm{e}-04$ & $1 \mathrm{e}-06$ & 0.07 & 0.00 & 24.03 \\
$1 \mathrm{e}-04$ & $1 \mathrm{e}-07$ & 0.06 & 0.00 & 22.69 \\
$1 \mathrm{e}-05$ & 0.1 & 0.26 & 0.48 & 339.58 \\
$1 \mathrm{e}-05$ & 0.01 & 0.08 & 0.70 & 3.76 \\
$1 \mathrm{e}-05$ & 0.001 & 0.03 & 0.00 & 3.08 \\
$1 \mathrm{e}-05$ & $1 \mathrm{e}-04$ & 0.02 & 0.00 & 9.44 \\
$1 \mathrm{e}-05$ & $1 \mathrm{e}-05$ & 0.04 & 0.00 & 20.32 \\
$1 \mathrm{e}-05$ & $1 \mathrm{e}-06$ & 0.06 & 0.00 & 20.51 \\
$1 \mathrm{e}-05$ & $1 \mathrm{e}-07$ & 0.05 & 0.00 & 23.05 \\
$1 \mathrm{e}-06$ & 0.1 & 0.26 & 0.50 & 747.09 \\
$1 \mathrm{e}-06$ & 0.01 & 0.08 & 0.77 & 3.58 \\
$1 \mathrm{e}-06$ & 0.001 & 0.03 & 0.00 & 3.15 \\
$1 \mathrm{e}-06$ & $1 \mathrm{e}-04$ & 0.02 & 0.00 & 8.63 \\
$1 \mathrm{e}-06$ & $1 \mathrm{e}-05$ & 0.05 & 0.00 & 12.25 \\
$1 \mathrm{e}-06$ & $1 \mathrm{e}-06$ & 0.05 & 0.00 & 22.47 \\
$1 \mathrm{e}-06$ & $1 \mathrm{e}-07$ & 0.05 & 0.00 & 22.01 \\
$1 \mathrm{e}-07$ & 0.1 & 0.26 & 0.31 & 135.87 \\
$1 \mathrm{e}-07$ & 0.01 & 0.08 & 0.73 & 3.33 \\
$1 \mathrm{e}-07$ & 0.001 & 0.03 & 0.00 & 3.09 \\
$1 \mathrm{e}-07$ & $1 \mathrm{e}-04$ & 0.02 & 0.00 & 8.16 \\
$1 \mathrm{e}-07$ & $1 \mathrm{e}-05$ & 0.05 & 0.00 & 22.77 \\
$1 \mathrm{e}-07$ & $1 \mathrm{e}-06$ & 0.05 & 0.00 & 25.65 \\
$1 \mathrm{e}-07$ & $1 \mathrm{e}-07$ & 0.05 & 0.00 & 27.85 \\
\hline & & & &
\end{tabular}

Table 2: Average results for MAFF over 100 Monte Carlo replicates, where the first two columns are control parameters of each method, $\mathcal{E}$ is the average error. CD is expressed as a rate and FD is the average number of points. 


\begin{tabular}{ccccr}
\hline$\lambda$ & $\xi$ & $\mathcal{E}$ & $\mathrm{CD}$ & \multicolumn{1}{c}{$\mathrm{FD}$} \\
\hline 0.6 & 0.1 & 0.56 & 0.32 & 0.07 \\
0.6 & 0.01 & 0.56 & 0.22 & 4.71 \\
0.6 & 0.001 & 0.56 & 0.00 & 2.30 \\
0.6 & $1 \mathrm{e}-04$ & 0.55 & 0.00 & 29.96 \\
0.6 & $1 \mathrm{e}-05$ & 0.62 & 0.01 & 265.87 \\
0.6 & $1 \mathrm{e}-06$ & 0.59 & 0.01 & 291.35 \\
0.6 & $1 \mathrm{e}-07$ & 0.61 & 0.00 & 309.84 \\
0.75 & 0.1 & 0.38 & 0.44 & 68.04 \\
0.75 & 0.01 & 0.36 & 0.50 & 4.06 \\
0.75 & 0.001 & 0.36 & 0.00 & 2.23 \\
0.75 & $1 \mathrm{e}-04$ & 0.38 & 0.00 & 26.90 \\
0.75 & $1 \mathrm{e}-05$ & 0.42 & 0.00 & 151.68 \\
0.75 & $1 \mathrm{e}-06$ & 0.42 & 0.00 & 195.25 \\
0.75 & $1 \mathrm{e}-07$ & 0.45 & 0.01 & 242.70 \\
0.8 & 0.1 & 0.33 & 0.40 & 67.94 \\
0.8 & 0.01 & 0.29 & 0.44 & 4.03 \\
0.8 & 0.001 & 0.29 & 0.00 & 2.12 \\
0.8 & $1 \mathrm{e}-04$ & 0.29 & 0.00 & 19.65 \\
0.8 & $1 \mathrm{e}-05$ & 0.36 & 0.00 & 142.96 \\
0.8 & $1 \mathrm{e}-06$ & 0.42 & 0.01 & 214.73 \\
0.8 & $1 \mathrm{e}-07$ & 0.41 & 0.00 & 211.68 \\
0.85 & 0.1 & 0.29 & 0.36 & 135.89 \\
0.85 & 0.01 & 0.22 & 0.63 & 3.80 \\
0.85 & 0.001 & 0.22 & 0.00 & 2.31 \\
0.85 & $1 \mathrm{e}-04$ & 0.27 & 0.00 & 23.87 \\
0.85 & $1 \mathrm{e}-05$ & 0.30 & 0.01 & 121.21 \\
0.85 & $1 \mathrm{e}-06$ & 0.33 & 0.00 & 191.14 \\
0.85 & $1 \mathrm{e}-07$ & 0.32 & 0.00 & 161.07 \\
0.9 & 0.1 & 0.27 & 0.32 & 0.05 \\
0.9 & 0.01 & 0.15 & 0.67 & 3.60 \\
0.9 & 0.001 & 0.15 & 0.00 & 2.57 \\
0.9 & $1 \mathrm{e}-04$ & 0.17 & 0.00 & 16.58 \\
0.9 & $1 \mathrm{e}-05$ & 0.25 & 0.01 & 105.92 \\
0.9 & $1 \mathrm{e}-06$ & 0.26 & 0.00 & 136.35 \\
0.9 & $1 \mathrm{e}-07$ & 0.30 & 0.00 & 149.92 \\
0.95 & 0.1 & 0.25 & 0.43 & 407.55 \\
0.95 & 0.01 & 0.10 & 0.76 & 3.37 \\
0.95 & 0.001 & 0.08 & 0.00 & 2.58 \\
0.95 & $1 \mathrm{e}-04$ & 0.11 & 0.00 & 12.84 \\
0.95 & $1 \mathrm{e}-05$ & 0.15 & 0.00 & 53.32 \\
0.95 & $1 \mathrm{e}-06$ & 0.18 & 0.00 & 75.07 \\
0.95 & $1 \mathrm{e}-07$ & 0.17 & 0.00 & 74.40 \\
0.99 & 0.1 & 0.25 & 0.45 & 475.43 \\
0.99 & 0.01 & 0.08 & 0.79 & 3.35 \\
0.99 & 0.001 & 0.03 & 0.00 & 2.78 \\
0.99 & $1 \mathrm{e}-04$ & 0.04 & 0.00 & 9.12 \\
0.99 & $1 \mathrm{e}-05$ & 0.07 & 0.00 & 27.86 \\
0.99 & $1 \mathrm{e}-06$ & 0.08 & 0.00 & 30.89 \\
0.99 & $1 \mathrm{e}-07$ & 0.08 & 0.00 & 30.53 \\
\hline & & & &
\end{tabular}

Table 3: Average results for MFFF over 100 Monte Carlo replicates, where the first two columns are control parameters of each method, $\mathcal{E}$ is the average error. CD is expressed as a rate and FD is the average number of points. 


\begin{tabular}{ccccc}
\hline$\lambda$ & $\xi$ & $\mathcal{E}$ & $\mathrm{CD}$ & $\mathrm{FD}$ \\
\hline 0.6 & 0.1 & 0.56 & 0.00 & 176.69 \\
0.6 & 0.01 & 0.59 & 0.02 & 264.79 \\
0.6 & 0.001 & 0.58 & 0.01 & 284.16 \\
0.6 & $1 \mathrm{e}-04$ & 0.57 & 0.00 & 301.95 \\
0.6 & $1 \mathrm{e}-05$ & 0.60 & 0.01 & 324.99 \\
0.6 & $1 \mathrm{e}-06$ & 0.58 & 0.02 & 282.45 \\
0.6 & $1 \mathrm{e}-07$ & 0.61 & 0.01 & 358.75 \\
0.75 & 0.1 & 0.42 & 0.00 & 148.67 \\
0.75 & 0.01 & 0.44 & 0.00 & 250.38 \\
0.75 & 0.001 & 0.49 & 0.01 & 287.51 \\
0.75 & $1 \mathrm{e}-04$ & 0.41 & 0.00 & 184.63 \\
0.75 & $1 \mathrm{e}-05$ & 0.42 & 0.00 & 236.80 \\
0.75 & $1 \mathrm{e}-06$ & 0.46 & 0.01 & 211.95 \\
0.75 & $1 \mathrm{e}-07$ & 0.45 & 0.02 & 201.07 \\
0.8 & 0.1 & 0.39 & 0.00 & 155.10 \\
0.8 & 0.01 & 0.43 & 0.00 & 229.62 \\
0.8 & 0.001 & 0.38 & 0.01 & 194.33 \\
0.8 & $1 \mathrm{e}-04$ & 0.43 & 0.01 & 243.41 \\
0.8 & $1 \mathrm{e}-05$ & 0.38 & 0.00 & 189.50 \\
0.8 & $1 \mathrm{e}-06$ & 0.39 & 0.00 & 200.82 \\
0.8 & $1 \mathrm{e}-07$ & 0.37 & 0.00 & 172.78 \\
0.85 & 0.1 & 0.28 & 0.00 & 118.21 \\
0.85 & 0.01 & 0.34 & 0.00 & 135.31 \\
0.85 & 0.001 & 0.34 & 0.00 & 183.92 \\
0.85 & $1 \mathrm{e}-04$ & 0.34 & 0.00 & 169.95 \\
0.85 & $1 \mathrm{e}-05$ & 0.37 & 0.00 & 231.49 \\
0.85 & $1 \mathrm{e}-06$ & 0.32 & 0.01 & 181.24 \\
0.85 & $1 \mathrm{e}-07$ & 0.37 & 0.00 & 214.87 \\
0.9 & 0.1 & 0.23 & 0.00 & 84.41 \\
0.9 & 0.01 & 0.27 & 0.00 & 152.84 \\
0.9 & 0.001 & 0.28 & 0.00 & 169.79 \\
0.9 & $1 \mathrm{e}-04$ & 0.24 & 0.00 & 123.97 \\
0.9 & $1 \mathrm{e}-05$ & 0.25 & 0.00 & 112.74 \\
0.9 & $1 \mathrm{e}-06$ & 0.29 & 0.00 & 169.13 \\
0.9 & $1 \mathrm{e}-07$ & 0.29 & 0.00 & 150.57 \\
0.95 & 0.1 & 0.15 & 0.00 & 54.82 \\
0.95 & 0.01 & 0.18 & 0.00 & 87.02 \\
0.95 & 0.001 & 0.18 & 0.00 & 77.35 \\
0.95 & $1 \mathrm{e}-04$ & 0.19 & 0.00 & 82.84 \\
0.95 & $1 \mathrm{e}-05$ & 0.18 & 0.00 & 82.65 \\
0.95 & $1 \mathrm{e}-06$ & 0.18 & 0.00 & 80.87 \\
0.95 & $1 \mathrm{e}-07$ & 0.17 & 0.00 & 72.61 \\
0.99 & 0.1 & 0.06 & 0.00 & 24.13 \\
0.99 & 0.01 & 0.08 & 0.00 & 29.55 \\
0.99 & 0.001 & 0.08 & 0.00 & 30.96 \\
0.99 & $1 \mathrm{e}-04$ & 0.08 & 0.00 & 35.79 \\
0.99 & $1 \mathrm{e}-05$ & 0.08 & 0.00 & 27.28 \\
0.99 & $1 \mathrm{e}-06$ & 0.08 & 0.00 & 34.42 \\
0.99 & $1 \mathrm{e}-07$ & 0.08 & 0.00 & 29.16 \\
\hline & & & &
\end{tabular}

Table 4: Average results for MFFFS over 100 Monte Carlo replicates, where the first two columns are control parameters of each method, $\mathcal{E}$ is the average error. CD is expressed as a rate and FD is the average number of points. 


\section{References}

Aggarwal, C. C. (2007). Data streams: models and algorithms, Volume 31. Springer Science \& Business Media.

Anagnostopoulos, C., D. K. Tasoulis, N. M. Adams, N. G. Pavlidis, and D. J. Hand (2012). Online linear and quadratic discriminant analysis with adaptive forgetting for streaming classification. Statistical Analysis and Data Mining 5(2), 139-166.

Balasubramanian, V., S.-S. Ho, and V. Vovk (2014). Conformal prediction for reliable machine learning: theory, adaptations and applications. Newnes.

Balzano, L., Y. Chi, and Y. M. Lu (2018). Streaming PCA and subspace tracking: The missing data case. Proceedings of the IEEE 106(8), 1293-1310.

Benczúr, A. A., L. Kocsis, and R. Pálovics (2018). Online machine learning in big data streams. arXiv preprint arXiv:1802.05872.

Bodenham, D. A. and N. M. Adams (2017). Continuous monitoring for changepoints in data streams using adaptive estimation. Statistics and Computing 27(5), 1257-1270.

Boutsidis, C., D. Garber, Z. Karnin, and E. Liberty (2015). Online principal components analysis. In Proceedings of the twenty-sixth annual ACM-SIAM Symposium on Discrete Algorithms, pp. 887-901. Society for Industrial and Applied Mathematics.

Bowers, K., V. Buscher, R. Dentten, M. Edwards, J. England, M. Enzer, A. K. Parlikad, and J. Schooling (2016). Smart infrastructure: Getting more from strategic assets. Centre for Smart Infrastructure and Construction.

Burnaev, E. and V. Vovk (2014). Efficiency of conformalized ridge regression. In Conference on Learning Theory, pp. 605-622.

Butler, L. J., N. Gibbons, P. He, C. Middleton, and M. Z. Elshafie (2016a). Evaluating the early-age behaviour of full-scale prestressed concrete beams using distributed and discrete fibre optic sensors. Construction and Building Materials 126, $894-912$.

Butler, L. J., N. Gibbons, P. He, C. Middleton, and M. Z. Elshafie (2016b). Evaluating the early-age behaviour of full-scale prestressed concrete beams using distributed and discrete fibre optic sensors. Construction and Building Materials 126 (Supplement C), $894-912$.

Butler, L. J., J. Xu, P. He, N. Gibbons, S. Dirar, C. R. Middleton, and M. Z. Elshafie (2018). Robust fibre optic sensor arrays for monitoring early-age performance of massproduced concrete sleepers. Structural Health Monitoring 17(3), 635-653.

Cardot, H. and D. Degras (2018). Online principal component analysis in high dimension: Which algorithm to choose? International Statistical Review 86(1), 29-50.

Champ, C. W. and W. H. Woodall (1987). Exact results for Shewhart control charts with supplementary runs rules. Technometrics 29(4), 393-399.

Chernozhukov, V., K. Wuthrich, and Y. Zhu (2018). Exact and robust conformal inference methods for predictive machine learning with dependent data. arXiv preprint arXiv:1802.06300.

Cleveland, W. S. (1979). Robust locally weighted regression and smoothing scatterplots. Journal of the American statistical association 74(368), 829-836.

Das, S., P. Saha, and S. Patro (2016). Vibration-based damage detection techniques used for health monitoring of structures: a review. Journal of Civil Structural Health Monitoring 6(3), 477-507.

Domingos, P. and G. Hulten (2003). A general framework for mining massive data streams. Journal of Computational and Graphical Statistics 12(4), 945-949.

Farrar, C. R. and K. Worden (2006). An introduction to structural health monitoring. Philosophical Transactions of the Royal Society A: Mathematical, Physical and Engineering Sciences 365(1851), 303-315. 
Gama, J. (2010). Knowledge discovery from data streams. CRC Press.

Giraud, L., J. Langou, and M. Rozloznik (2005). The loss of orthogonality in the gramschmidt orthogonalization process. Computers $\&$ Mathematics with Applications $50(7)$, 1069-1075.

Glisic, B., D. Inaudi, J. M. Lau, Y. C. Mok, and C. T. Ng (2005). Long-term monitoring of high-rise buildings using long-gauge fibre optic sensors. In 7th International Conference on Multi-Purpose High-Rise Towers and Tall Buildings, Dubai, UAM, 10 - 11 December (on conference $C D$, paper \#0416).

Goodfellow, I., Y. Bengio, and A. Courville (2016). Deep learning. MIT press.

Haykin, S. S. (2008). Adaptive filter theory. Pearson.

Hernandez-Garcia, M. R. and S. F. Masri (2014). Application of statistical monitoring using latent-variable techniques for detection of faults in sensor networks. Journal of Intelligent Material Systems and Structures 25(2), 121-136.

Johnstone, I. M. (2001). On the distribution of the largest eigenvalue in principal components analysis. Annals of Statistics 29(2), 295-327.

Jolliffe, I. (2011). Principal component analysis. Springer.

Kim, A. Y., C. Marzban, D. B. Percival, and W. Stuetzle (2009). Using labeled data to evaluate change detectors in a multivariate streaming environment. Signal Processing 89(12), 2529-2536.

Lau, F. D.-H., N. M. Adams, M. A. Girolami, L. J. Butler, and M. Z. E. B. Elshafie (2018). The role of statistics in data-centric engineering. Statistics $\&$ Probability Letters 136, $58-62$.

Lau, F. D.-H., L. J. Butler, N. M. Adams, M. Z. E. B. Elshafie, and M. A. Girolami (2018). Real-time statistical modelling of data generated from self-sensing bridges. Proceedings of the Institution of Civil Engineers - Smart Infrastructure and Construction 171(1), $3-13$.

Laxhammar, R. and G. Falkman (2015). Inductive conformal anomaly detection for sequential detection of anomalous sub-trajectories. Annals of Mathematics and Artificial Intelligence 74(1-2), 67-94.

Lei, J., M. G'Sell, A. Rinaldo, R. J. Tibshirani, and L. Wasserman (2018). Distributionfree predictive inference for regression. Journal of the American Statistical Association 113(523), 1094-1111.

Lei, J., A. Rinaldo, and L. Wasserman (2015). A conformal prediction approach to explore functional data. Annals of Mathematics and Artificial Intelligence 74(1-2), 29-43.

Lei, J., J. Robins, and L. Wasserman (2013). Distribution-free prediction sets. Journal of the American Statistical Association 108(501), 278-287.

Lei, J. and L. Wasserman (2014). Distribution-free prediction bands for non-parametric regression. Journal of the Royal Statistical Society: Series B (Statistical Methodology) $76(1), 71-96$.

Measures, R. M., M. LeBlanc, K. Liu, S. Ferguson, T. Valis, D. Hogg, R. Turner, and K. McEwen (1992). Fiber optic sensors for smart structures. Optics and Lasers in Engineering 16(2), 127-152.

Mezzadri, F. (2006). How to generate random matrices from the classical compact groups. arXiv preprint math-ph/0609050.

Mitliagkas, I., C. Caramanis, and P. Jain (2013). Memory limited, streaming PCA. In Advances in Neural Information Processing Systems, pp. 2886-2894.

Nadler, B. (2011). On the distribution of the ratio of the largest eigenvalue to the trace of a Wishart matrix. Journal of Multivariate Analysis 102(2), 363-371.

Novembre, J. and M. Stephens (2008). Interpreting principal component analyses of spatial population genetic variation. Nature Genetics 40(5), 646-649. 
Oja, E. (1992). Principal components, minor components, and linear neural networks. Neural Networks 5(6), 927-935.

Oja, E. and J. Karhunen (1985). On stochastic approximation of the eigenvectors and eigenvalues of the expectation of a random matrix. Journal of Mathematical Analysis and Applications 106(1), 69-84.

Micron Optics (2013). ENLIGHT User Guide. http://www . micronoptics . com/download/ enlight-user-guide-revision-1-138/\#. Accessed: 2019-04-06.

Sanger, T. D. (1989). Optimal unsupervised learning in a single-layer linear feedforward neural network. Neural Networks 2(6), 459-473.

Scholz, M. (2007). Analysing periodic phenomena by circular PCA. In International Conference on Bioinformatics Research and Development, pp. 38-47. Springer.

Stewart, G. W. (1980). The efficient generation of random orthogonal matrices with an application to condition estimators. SIAM Journal on Numerical Analysis 17(3), 403409.

Todd, M. D., J. M. Nichols, S. T. Trickey, M. Seaver, C. J. Nichols, and L. N. Virgin (2006). Bragg grating-based fibre optic sensors in structural health monitoring. Philosophical Transactions of the Royal Society A: Mathematical, Physical and Engineering Sciences 365(1851), 317-343.

Vovk, V. (2013). Conditional validity of inductive conformal predictors. Machine Learning 92(2-3), 349-376.

Vovk, V., A. Gammerman, and G. Shafer (2005). Conformal prediction. Springer.

Vovk, V., I. Nouretdinov, and A. Gammerman (2009). On-line predictive linear regression. The Annals of Statistics 37(3), 1566-1590.

Warmuth, M. K. and D. Kuzmin (2008). Randomized online PCA algorithms with regret bounds that are logarithmic in the dimension. Journal of Machine Learning Research 9(Oct), 2287-2320.

Weng, J., Y. Zhang, and W.-S. Hwang (2003). Candid covariance-free incremental principal component analysis. IEEE Transactions on Pattern Analysis and Machine Intelligence 25(8), 1034-1040.

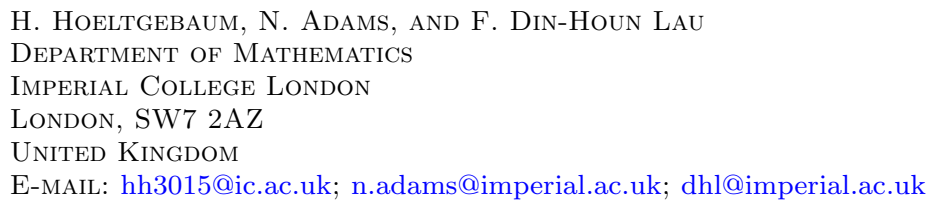

